\title{
Systemic impact of the expression of the mitochondrial alternative oxidase on Drosophila
}

\section{development}

André F. Camargo ${ }^{1}$, Sina Saari ${ }^{2}$, Geovana S. Garcia ${ }^{1}$, Marina M. Chioda ${ }^{1}$, Murilo F. Othonicar ${ }^{1}$, Ailton A. Martins ${ }^{1}$, Gabriel Hayashi ${ }^{1}$, Johanna ten Hoeve ${ }^{3}$, Howard T. Jacobs ${ }^{2}$, Daniel G.

Pinheiro $^{1}$, Eric Dufour ${ }^{2} \&$ Marcos T. Oliveira ${ }^{1, *}$

${ }^{1}$ Departamento de Tecnologia, Faculdade de Ciências Agrárias e Veterinárias de Jaboticabal, Universidade Estadual Paulista "Júlio de Mesquita Filho", Jaboticabal, SP, Brazil

${ }^{2}$ Faculty of Medicine and Health Technology, Tampere University, Tampere, Finland

${ }^{3}$ UCLA Metabolomics Center, Department of Molecular and Medical Pharmacology, University of California Los Angeles, Los Angeles, CA, USA

${ }^{*}$ Corresponding author: Via de Acesso Prof. Paulo Donato Castellane, s/n, Departamento de Tecnologia, FCAV/UNESP, Jaboticabal, SP 14884-900, Brazil; telephone: +55 (16) 3209-7536; e-mail: marcos.t.oliveira@unesp.br 


\section{Abstract}

Despite the beneficial effects shown when the mitochondrial alternative oxidase AOX from Ciona intestinalis (Tunicata: Ascidiacea) is xenotopically expressed in mammalian and insect models, important detrimental outcomes have also been reported, raising concerns regarding its envisioned deployment as a therapy enzyme for human mitochondrial and related diseases. Because of its non-proton pumping terminal oxidase activity, AOX can bypass the cytochrome c segment of the respiratory chain and alleviate the possible overload of electrons that occurs upon oxidative phosphorylation (OXPHOS) dysfunction, not contributing though to the proton-motive force needed for mitochondrial ATP synthesis. We have shown previously that AOX-expressing flies present a dramatic drop in pupal viability when the larvae are cultured on a low nutrient diet, indicating that AOX interferes with normal developmental metabolism. Here, we applied combined omics analyses to show that the interaction between low nutrient diet and AOX expression causes a general alteration of larval amino acid metabolism and lipid accumulation, which are associated with functional and morphological alterations of the larval digestive tract and with a drastic decrease in larval biomass accumulation. Pupae at the prelethality stage present a general downregulation of mitochondrial metabolism and a signature for starvation and deregulated signaling processes. This AOX-induced lethality is partially rescued when the low nutrient diet is supplemented with tryptophan and/or methionine. The developmental dependence on these amino acids, associated with elevated levels of lactate dehydrogenase, lactate, 2-hydroxyglutarate, choline-containing metabolites and breakdown products of membrane phospholipids, indicates that AOX expression promotes tissue proliferation and growth of the Drosophila larvae, but this is ultimately limited by energy 
dissipation via mitochondrial uncoupling. We speculate that the combination of diet and AOX expression may be used for the metabolic regulation of proliferative tissues, such as tumors.

Key words: mitochondria, amino acid metabolism, nutrient deprivation, oxidative phosphorylation 


\section{Introduction}

Although mitochondria have many important roles in eukaryotic cells, the bulk production of ATP via oxidative phosphorylation (OXPHOS) is traditionally viewed as one of their main contributing processes for cellular function. Several mitochondrial inner membraneembedded enzymes, such as NADH dehydrogenase (or complex I, CI), succinate dehydrogenase (or complex II, CII), and mitochondrial glycerol-3-phosphate dehydrogenase (mGPDH), among others, may convergently initiate OXPHOS by catalyzing the reduction of coenzyme Q $\left(\mathrm{CoQ} / \mathrm{CoQH}_{2}\right)$. These reactions are coupled with the reoxidation of metabolites/electron carriers needed in the mitochondrial matrix or outside mitochondria for the continuation of diverse pathways involved in the partial or complete oxidation of nutrients, such as monosaccharides, amino acids, and fatty acids. Starting a serial chain of redox reactions that we herein refer to as the cytochrome $c$ segment, the cytochrome $b c_{1}$ complex (or complex III, CIII) oxidizes $\mathrm{CoQH}_{2}$, reducing cytochrome $c$, which in turn is oxidized by cytochrome $c$ oxidase (or complex IV, CIV) in a reaction that uses molecular oxygen $\left(\mathrm{O}_{2}\right)$ as the final acceptor of electrons and produces $\mathrm{H}_{2} \mathrm{O}$. The electron transfer activities of CI, III and IV, are paired with the pumping of protons from the mitochondrial matrix into the intermembrane space. The potential energy of the electrochemical gradient formed is eventually used by the ATP synthase to phosphorylate ADP, as the enzyme allows the return of the protons to the matrix.

Notably, the electrochemical gradient, the production of ATP, and the reoxidation of electron carriers are crucial for the regulation of the whole cellular metabolism. OXPHOS function directly affects the catabolic and anabolic functions of the tricarboxylic acid (TCA) cycle, depending on factors such as tissue/cell type, stage of development, temperature and/or nutritional availability, among others. For example, in proliferative tissues such as in tumors or 
those in the growing Drosophila larvae, a significant fraction of the TCA cycle intermediates are diverted and used as precursors for the synthesis of lipids, nucleotides, carbohydrates and proteins, a process generally referred to as cataplerosis ${ }^{1}$. Although cataplerosis appears to be the most important function of mitochondria during biomass accumulation, at least in some cases it may not proceed without $\mathrm{CoQH}$ 2 reoxidation by functional mitochondrial electron transfer complexes $^{2,3}$. In addition, OXPHOS dysfunction is related to several human disease conditions, and may be the cause for myriad types of mitochondrial diseases, whose onset and severity vary broadly, and most of which still do not have effective treatment ${ }^{4}$.

Protists, fungi, plants, and most metazoans, but not vertebrates and insects, have an OXPHOS system with a second terminal oxidase, the non-proton-pumping alternative oxidase $(\mathrm{AOX})^{5}$. AOX activity bypasses CIII/CIV by directly coupling $\mathrm{O}_{2}$ reduction with $\mathrm{CoQH}_{2}$ reoxidation. Although this is not coupled to ATP synthesis, AOX function has been associated with maintenance of electron flow and cellular redox potential, and with counteracting excess reactive oxygen species (ROS) that are produced in cases of OXPHOS stress, overload or blockage $^{3,6-8}$, conditions that are similar to those found in cells of human patients with mitochondrial diseases. The premise that the transgenic expression of AOX would be beneficial for higher animals under OXPHOS dysfunction has been successfully tested in diverse models ${ }^{9-}$ ${ }^{14}$. In addition to providing respiration resistant to inhibitors of CIII and CIV, AOX enabled complete or partial recovery of deleterious phenotypes caused by mutations in subunits of the OXPHOS complexes ${ }^{3,10,15-17}$ and has been considered as a therapy enzyme in putative future treatments ${ }^{18,19}$.

Nevertheless, AOX expression studies in model organisms has been limited, so the alterations it may cause to normal metabolism and physiology are not fully understood ${ }^{20-23}$, 
especially considering that the action of the enzyme could uncouple mitochondria and generate heat. We have been using Drosophila melanogaster lines expressing AOX from the tunicate Ciona intestinalis (Ascidiacea) to test possible limitations in the use of this enzyme in higher animals and to understand how we can counterbalance these limitations. To date, fly reproduction and development appear to be negatively affected by AOX. We have shown that AOX expressed constitutively under the control of the tubulin promoter (tubAOX) causes a dosedependent depletion of mature spermatozoids, which does not cause sterility, but leads to a dramatic disadvantage for AOX-nonexpressing males in sperm competition assays ${ }^{24}$. The larvae of the same fly lines, however, are resistant to extreme cold stress, presenting faster developmental time, higher viability ${ }^{22}$ and higher motility ${ }^{25}$ at temperatures as low as $12^{\circ} \mathrm{C}$.

We also recently reported that AOX expression heavily compromises adult eclosion when the flies are cultured on a low nutrient (LN) $\operatorname{diet}^{22}$. This pupal lethality is caused by strong ubiquitous expression of AOX, using the efficient, well stablished bipartite $U A S-A O X / d a G A L 4$ expression system ${ }^{10}$. Our LN diet is a simple food in which the only source of nutrients, $3.5 \%$ yeast extract $(\mathrm{w} / \mathrm{v})$, is sufficient for AOX-nonexpressors to reach the adult stage. Our standard (SD) laboratory diet, on the other hand, contains $1.5 \%$ sucrose, $3 \%$ glucose, $1.5 \%$ maize flour, $1 \%$ wheat germ, $1 \%$ soy flour, and $3 \%$ molasses, in addition to $3.5 \%$ yeast extract $(\mathrm{w} / \mathrm{v})$. Although extensive work has been performed with dietary supplementation using sugars, vitamins, minerals, extra yeast extract, and other simple compounds, only nutritionally and structurally complex ingredients of the SD diet, such as molasses, could in fact rescue the lethality of AOX-expressing flies cultured on $\mathrm{LN}^{23}$. Mass spectrometry revealed a complex array of metabolites in a fraction of molasses that is rich in intermediates of the TCA cycle, among other molecules, but no compound that have been tested to date could individually rescue the 
lethality of AOX-expressing flies cultured on LN. This illustrates the complexity of the metabolic rearrangement that AOX may cause, which was first suggested when AOX failed to rescue the deleterious phenotype of well-established mitochondrial mutants ${ }^{21,26-28}$, and yet it was effective against genetic defects with no apparent direct link to mitochondria ${ }^{29}$.

To gain more insights into the molecular basis of the fatal developmental interaction between AOX expression and LN diet, and possibly how to prevent it, here we applied omics approaches to infer the metabolic changes occurring in AOX-expressing larvae and pupae cultured on SD or LN diets. Our data suggests that strong AOX expression promotes a rearrangement in larval amino acid metabolism that is in contrast to the changes induced by the LN diet. This rearrangement is accompanied by increased larval feeding behavior, functional and morphological alterations of the larval intestines, impaired nutrient storage in the larvae, and signs of starvation and deregulated signaling processes in the pupae. Pupal lethality can be partially rescued by dietary supplementation of the feeding larvae with methionine or tryptophan, suggesting an important role for the one-carbon metabolism in the adaptive processes that allow AOX-expressing flies to complete development.

\section{Results and Discussion}

Strong ubiquitous expression of AOX causes systemic developmental failure on low nutrient diet

To verify which levels of ubiquitously-driven AOX expression cause pupal lethality when the larvae are cultured on LN diet, we performed developmental assays similar to those shown in our previously published data ${ }^{22}$. This time we included additional $U A S-A O X$ lines that, when crossed with the $d a G A L 4$ driver line, provided flies with ubiquitous levels of AOX expression assigned as "weak", "intermediate" and "strong". Once again, we found that the pupal 
lethality only appears when strong expression levels of the enzyme are combined with LN diet

(Figure 1A). We confirmed that LN diet itself does not affect AOX transcript or protein levels

(Figure 1B,C). We then tested if the lethality of these AOX-expressing flies in LN diet is caused by the cellular metabolic disturbances that occur in a specific tissue, resulting in failure of adult eclosion. We drove strong tissue-specific expression of AOX in three larval tissues of main importance for whole animal energy metabolism, and checked if this would reproduce the typical pupal lethality observed when using the ubiquitous $d a G A L 4$ driver $^{22,23}$. Figure 1D shows that strong expression of AOX in the larval musculature, the nervous system, or in the fat body, in combination with LN diet, had no effect on pupal viability. This suggests that the lethal phenotype described above is a consequence of AOX expression in a less obvious tissue, or of a systemic metabolic derangement initiated in multiple tissues.

\section{Strong ubiquitous expression of AOX induces functional and morphological changes in the} larval digestive system

Instead of incessantly testing any other of the innumerous GAL4 driver lines available, either individually or in combination, we decided to evaluate the putative systemic metabolic derangement by performing deep RNA sequencing (RNA-seq) of whole individuals with strong expression of AOX. This could allow us to identify general and tissue-specific transcriptional trends caused by LN diet and by AOX expression, which combined would lead to pupal lethality. Although this approach would not reveal spatial gene misexpression, it could help us hypothesize scenarios indicative of tissue-specific alterations. We selected wandering third-instar (L3) larvae and pre-pharate pupae to obtain insights into the immediate post-feeding and pre-mortem stages, respectively. General analyses of the data either separately (Supplemental Tables S1-S6) or 
combined (Supplemental Tables S7-S8) showed that the transcriptomic changes from larva to pre-pharate pupa, independent of diet and AOX expression, were as expected and previously described $^{30,31}$. The pupae present a general upregulation of mitochondrial and nervous system genes, which is consistent with the switch from a primarily glycolytic to an OXPHOS-based metabolism from larva to adult ${ }^{32,33}$, and with the morphogenesis of the adult brain and eyes. We also observed downregulation of genes involved with proteolytic/autophagic processes, information pathways/cell cycle, sugar transport and metabolism, and genes encoding alkaline phosphatases, which likely reflects the end of the metamorphic phase. Remodeling of the epidermis/cuticle/exoskeleton is also apparent by the up and downregulation of different groups of genes (Supplemental Tables S1-S8), which probably reflect the cell migration events that ensure dorsal thoracic and abdominal midline closure during metamorphosis ${ }^{34}$.

In wandering L3 larvae, a multifactorial analysis revealed that the LN diet is responsible for the largest change in the fly transcriptome, with significant alterations in the levels of almost $10 \%$ of all the 13809 unique transcripts identified (Supplemental Table S9). On the other hand, strong AOX expression caused alterations in the levels of approximately $2 \%$ of all these transcripts (Supplemental Table S10), and the interaction between LN and AOX (LN-AOX) altered further 2\% (Supplemental Table S11). Based on enrichment analysis and functional annotation, transcripts related to metabolic processes and nutrient transport were extensively affected by the LN diet, as expected, in addition to genes grouped in smaller clusters assigned as sexual maturation, RNA binding, and protein folding (Supplemental Figure S1). Interestingly, all three factors, LN diet, AOX expression, and LN-AOX interaction, presented two common gene clusters: proteolysis and chitin metabolism (Figure 2). We surveyed the data from modENCODE ${ }^{30,35}$ deposited in Flybase (flybase.org) to map the tissues in which the transcripts 
in these two clusters are usually present at high abundance. The proteolysis and chitin metabolism transcripts altered by LN diet (Figure 2A) appear to be scattered among different organs, and not to be overrepresented in a particular larval tissue (Supplemental Table S12). However, for AOX expression (Figure 2B) and the LN-AOX interaction (Figure 2C), we observed that the transcripts are commonly abundant in tissues related to larval digestion (the digestive system and the salivary glands, Supplemental Table S13 and S14). Interestingly, when we analyzed the transcripts in all clusters in the AOX and the LN-AOX groups, 63 and 58\% respectively are usually associated with digestive tissues, according to the data deposited in Flybase. Transcripts related to larval/adult digestion in LN diet account for only about $20 \%$ of total clustered transcripts in our analysis (Supplemental Tables S12-S14).

Endo/exo peptidases are one of the largest families of digestive enzymes in flies ${ }^{36}$, making up the majority of genes in the proteolysis clusters we identified. Flies also express a variety of chitinases and glucanases that supposedly play a role on yeast cell digestion, which would be especially important in our LN diet that contains only yeast extract as source of nutrients ${ }^{22}$. The genes in the chitin metabolism clusters may also be important for the development and function of the internal lining of the intestines, which consists primarily of epithelial cells containing chitin-based structures that must frequently be remodeled to allow proper digestion and insect growth ${ }^{37}$. Although the gastrointestinal tract of Drosophila is a complex organ with multiple cell types of different developmental origin ${ }^{38}$, our data suggests that in the immediate post-feeding larvae, before the animals enter the pupa stage during which they must rely on stored nutrients to complete the costly processes involved with metamorphosis, strong ubiquitous AOX expression causes a significant alteration of the larval digestion and nutrient absorption. To test this hypothesis, we first checked the intestinal morphology of our 
larvae and noticed that $\mathrm{LN}$ diet causes shortening of the intestines in the control flies, and that

strong AOX expression prevents this shortening from happening (Figure 3A). We next tested if strong expression of AOX throughout development using an intestine-specific GAL4 driver would reproduce the pupal lethality observed with the ubiquitous expression system. We observed that the pupal viability rates in AOX-expressors were as high as in the AOXnonexpressing controls (Figure 3B). These findings again indicate that the AOX-induced pupal lethality is caused by a systemic metabolic alteration involving multiple tissues/organs, among which the digestive tract is significantly affected.

\section{Strong ubiquitous expression of AOX leads to decreased larval biomass accumulation}

Functional and morphological changes in the larval intestines as a consequence of a systemic metabolic alteration might indicate that strong ubiquitous expression of AOX disturbs larval food consumption and/or biomass storage. We first examined food consumption in the early L3 stage and in fact observed that AOX-expressing larvae eat more, independently of diet (Figure 3C). However, the increased feeding behavior is not reflected as gain in larval weight. We observed that strong AOX expression in SD diet causes a drop in body mass equivalent to that caused by the LN diet in control larvae $(\sim 15 \%)$, but these conditions individually are not sufficient to cause detriments to the fly development. The effects of the two conditions combined were additive: we verified an almost $40 \%$ decrease in the body mass of wandering L3 larvae of the LN-AOX group (Figure 4A-C). The drop in larval body mass is accompanied by a significant decrease in larval and pupal size, independent of sex (Supplemental Figure S4). Interestingly, we have previously reported that adult flies expressing AOX with the same $U A S / G A L 4$ system, cultured on SD diet, present a significantly more pronounced loss of weight as the flies age ${ }^{10}$. 
Although AOX-expressing larvae consume more food, the decreased biomass accumulation suggests that when cultured on LN diet, they fail to store enough nutrients before entering the pupa stage. We have shown previously that larval triglycerides are significantly lower in larvae cultured on LN, and that strong AOX expression had no significant effect on the levels of these storage molecules ${ }^{23}$. Because in Drosophila triglyceride levels increase as the larvae grow, and continue increasing during metamorphosis at the expense of membrane lipids ${ }^{39}$, we quantitated total fat content in our larvae by ether extraction to get an estimate for combined triglycerides, membrane lipids, and other lipids. LN diet lowered total larval fat, which is consistent with the drop in triglycerides previously seen ${ }^{23}$. Interestingly, we also detected a tendency for AOX-expressing larvae cultured on SD to accumulate more fat than the other larvae (Figure 4D), which we speculate may be due to elevated membrane lipid accumulation, since triglycerides are unchanged under this condition ${ }^{23}$. The larval transcriptome indicates that the LN diet causes a substantial change in lipid metabolism (Supplemental Figure S1), of which fatty acid $\beta$-oxidation appears appreciably downregulated (Supplemental Figure S5), and both LN diet and LN-AOX cause a significant rearrangement in the levels of membrane proteins (Supplemental Figures S1 and S3). Surprisingly, LN-AOX pupae present elevated transcript levels of three enzymes involved in triglyceride biosynthesis (Supplemental Figure S5), suggesting deregulated lipid metabolism during metamorphosis.

We also estimated total content of larval protein, as this is another stored nutrient important for pupal development, and observed that strong ubiquitous expression of AOX, irrespective of diet, causes a $25-40 \%$ increase in larval protein levels (Figure 4E). This is consistent with the increased feeding behavior (Figure 3C), and also with the changes in protein metabolism detected in the transcriptome (Figure 2) and in amino acid levels described below 
(see next sections for details). Taken together, our data indicates that AOX-expressing larvae accumulate more protein and more fat (likely as membrane lipids) per larval mass. The LN diet, in which yeast extract is the sole nutrient source, appears to prevent fat accumulation, still providing enough nutrients for protein biosynthesis. We, therefore, speculate that the changes observed in the larval intestines and in feeding behavior are likely due to altered nutrient absorption, in an attempt for the AOX larvae to reach its necessary, increased nutrient stores.

\section{The interaction between strong ubiquitous expression of AOX and low nutrient diet causes} developmental arrest due to pupal starvation

The increased protein and fat content in SD-cultured, AOX-expressing larvae may be a compensatory physiological adaptation that allows the AOX-expressing flies to go through metamorphosis and reach the adult phase. The pupal tissues, therefore, may likely have additional nutrients to support all metamorphic processes and balance the energy dissipation that is putatively caused by AOX activity in the mitochondrial electron transfer system. We next investigated the pre-pharate transcriptional landscape to gain insight into the molecular events preceding the lethality of LN-AOX flies. Our multifactorial analysis revealed that strong AOX expression induces a change in only $0.8 \%$ of all 13,687 transcripts identified (Supplemental Table S16), with minor functional clusters detected (Supplemental Figure S6B), consistent with the idea of a physiological larval adaptation that has discreet impact on pupal development and survival. The LN diet affected $1.9 \%$ of all pupal transcripts (Supplemental Table S15), of which the major functional clusters were again proteolysis and chitin metabolism, in addition to tissue integrity and transcriptional regulation (Supplemental Figure S6A). With further 5.4\% of transcriptomic changes caused by the LN-AOX interaction (Supplemental Table S17), the major 
transcript clusters in this condition were assigned as stress response, transcriptional regulation, and tissue integrity and stimulus response (Supplemental Figure S7). Because LN and LN-AOX presented each a mitochondrial cluster containing transcripts of several OXPHOS subunits (Supplemental Figures S6A and S7) and because changes in nutrient storage may directly alter OXPHOS output, we analyzed in more details the transcripts coding for all subunits of the OXPHOS complexes by manual clustering. We observed a general upregulation of OXPHOS transcripts in the LN-cultured, control flies (Figure 5A), which we infer leads to higher efficiency in ATP production upon lower nutrient availability. Conversely, AOX induces downregulation of OXPHOS transcripts in both diets, but the effect is more pronounced in LNAOX pupae (Figure 5A), which also presented $>12$ fold higher levels of lactate dehydrogenase (Ldh) transcripts (Supplemental Table S17). High Ldh and low OXPHOS levels suggest that the LN-AOX interaction causes mitochondrial dysfunction or metabolic rerouting during a developmental step that crucially relies on oxidative metabolism.

Judged by the stress response indicators (Supplemental Figure S7), it is possible that at this particular time in development, our LN-AOX pupae had already consumed almost all nutrients stored by the larvae (of which fat appears to be limiting, Figure 4D), which may suggest that the observed OXPHOS downregulation and most other transcriptomic changes are a consequence of starvation. We first noted that the transcript levels of Insulin-like receptor (InR) were $\sim 3.2$ fold higher in LN-AOX pupae, and then we manually clustered the transcripts of other genes associated with the Insulin/Insulin-like growth factor-1 Signaling (IIS) pathway or of known genes involved in starvation-mediated response (Figure 5B). Most of these were upregulated in LN-AOX pupae, especially ImpL2 (elevated $>10$ fold), a homolog of the human insulin-like growth factor binding protein-7 (IGFBP-7) gene, whose product is a secreted factor 
that binds the Drosophila insulin-like peptide (Ilp) 2 and inhibits growth upon nutritional deprivation $^{40}$. Another growth inhibitor highly expressed in LN-AOX pupae is $\operatorname{Ilp} 8(\sim 21$ fold higher); its encoded peptide is produced by the imaginal discs to delay pupariation in larvae ${ }^{41,42}$ and arrest metamorphosis in early pupae ${ }^{43}$. In both cases, Ilp 8 inhibits ecdysone biosynthesis by the prothoracic gland when the imaginal discs have abnormal developmental growth. Ilp 8 transcripts were also elevated in LN-AOX larvae ( $\sim 2.3$ fold, not statistically significantly, though) (Figure S8), but this is clearly not enough to prevent production of sufficient levels of ecdysone to induce pupariation.

Upregulation of Ilp8 in imaginal discs appears to be regulated by the Jun N-terminal Kinase (JNK) signaling pathway ${ }^{41-43}$. AOX expression in the fly thoracic dorsal epithelium during metamorphosis can rescue the downregulation of the JNK pathway ${ }^{29}$, which in turn can also be activated upon loss of tissue integrity ${ }^{44}$. Moreover, JNK signaling activates the cell proliferation-promoting JAnus Kinase (JAK)/Signal Transducer and Activator of Transcription (STAT) signaling cascade in diverse tissues via upregulation of the cytokines Upd, Upd2 and Upd $3^{44-47}$. We observed that our LN-AOX pupae also presented a general upregulation of the JNK and JAK/STAT signaling. Particularly, the transcript levels of upd2 and upd3, and of their receptor dome were elevated $\sim 24, \sim 46$ and 2.3 fold, respectively (Figure 5B). Interestingly, diverse cell signaling-related genes are represented in the largest cluster of altered transcripts in the LN-AOX larva transcriptome (Supplemental Figure S3). In addition, strong AOX expression in larvae causes a significant increase in the levels of upd2 and upd3 (Supplemental Figure S8), but their levels are normalized in SD-cultured pupae (Figure 5B), indicating that the JAK/STAT signaling might have a role in the physiological adaptation that the larvae undergo in the presence of AOX. Considering the contrasting effects on larval fat storage caused by strong 
ubiquitous AOX expression and LN diet, our data suggests that most LN-AOX individuals "run out of fuel" during metamorphosis, with concomitant alterations in the IIS, JNK and JAK/STAT signaling.

Partial rescue of $A O X$-induced pupal lethality by methionine and/or tryptophan supplementation

Because the changes in larval metabolism induced by strong expression of AOX appear to be key in our understanding of the pupal lethality in LN diet, we performed an additional omics approach and investigated the whole-body metabolome of AOX-expressing and control larvae cultured on SD and LN diets. A multifactorial analysis revealed that the LN diet causes significant alterations in the levels of $45 \%$ of the 113 unique metabolites identified (Supplemental Table S18). As expected, we observed that the LN diet promotes decreased levels of most intermediates of the TCA cycle and of the pentose phosphate pathway, and a general rearrangement in the levels of nitrogenous bases, nucleosides and nucleotides, including an increase in markers of energy depletion such as nucleotide monophosphates and NAD+ (Figure 6). The observed changes in these metabolites are consistent with a combination of high energy demand for biosynthesis and storage of new biomolecules (characteristic of the proliferative nature of larval tissues) and low supply of high energy nutrients from the diet.

AOX expression, on the other hand, led to increased levels of most TCA cycle intermediates (Figure 6), pointing to important modifications of mitochondrial metabolism that were not evident from the larval transcriptomic analyses. Succinate is the only detected intermediate not elevated, so we speculate that it (and/or succinyl-CoA, which was not detected in our analysis) might be cataplerotically deviated from the TCA cycle upon accumulation of the other intermediates. Although succinate/succinyl-CoA can have diverse other roles in multiple 
pathways $^{48}$, an association can be made with the methionine cycle, of which several metabolites were elevated due to AOX expression (Figure 6). The methionine cycle is key in the cellular production of the universal methyl donor $S$-adenosylmethionine (SAM), but most importantly, it is interconnected with the metabolism of several other amino acids and nucleotides needed during tissue proliferation ${ }^{49,50}$. The amino acids serine, glycine and tryptophan, among others, can fuel the folate cycle, which in turn provides methyl groups via 5-methyl-tetrahydrofolate (5methyl-THF) to the methionine cycle; together, both cycles are referred to as the one-carbon metabolism ${ }^{51-53}$. Tryptophan, serine, glycine and two of the precursors in the serine de novo biosynthesis pathway, 3-phosphoglycerate (3-PG, possibly diverted from glycolysis/gluconeogenesis) and 3-phosphoserine (3-PS), were also elevated due to AOX expression (glycine not statistically significant, though) (Figure 6).

Consistent with the significant number of proteolysis and amino acid metabolism transcripts that were altered by AOX and LN diet in larvae, we in fact observed a substantial rearrangement in the levels of many other amino acids in the larvae under these conditions (Figure 6). Interestingly, we have previously tested titrating yeast extract up to $10 \%(\mathrm{w} / \mathrm{w})$ into the LN diet to provide extra dietary protein, but we observed no improvement in pupal viability upon high levels of ubiquitously-driven AOX expression ${ }^{23}$. We then speculated whether yeast extract only does not contain a balanced proportion of amino acids for the proper development of AOX-expressing flies. Although rich in proteins, yeast extract generally contains proportionally low levels of the essential methionine and tryptophan, and the non-essential cysteine ${ }^{54}$. Accordingly, the larval metabolome showed decreased levels of methionine and tryptophan due to $\mathrm{LN}$ diet. AOX expression, on the other hand, caused a significant increase in the levels of these amino acids, and of serine, glycine, 3-PG and 3-PS, but this pattern is either not present or 
not as pronounced in the metabolome of LN-AOX larvae (Figure 6). Remarkably, methionine and tryptophan, among other essential (arginine and valine) and non-essential (glutamine, cysteine and aspartate) amino acids, were enriched in the water-soluble fraction of molasses that, when added to the LN diet, rescued the lethal phenotype caused by the LN-AOX interaction ${ }^{23}$.

We therefore tested the supplementation of the SD and LN diets with extra methionine and tryptophan, individually and in combination, and observed a significant, albeit partial, rescue of the viability (Figure 7A) and body size (Supplemental Figure S4B-E) of AOX-expressing pupae cultured on LN diet. The effects were more apparent with lower concentrations of these amino acids, and were not additive, confirming that these flies do require a proper balance of these molecules for development, and suggesting that these two amino acids are contributing to the same pathway. To check if addition of any other amino acid would also rescue the AOXdriven pupal lethality, we also tested the supplementation of extra proline and/or glutamate to the LN diet, but these failed to promote any rescue (Figure 7B). Proline and glutamate oxidation would also provide reducing power for ATP synthesis via OXPHOS, so our results suggest that this may not be a limiting factor for LN-AOX flies, as previously indicated by addition of extra sugar to the $\mathrm{LN} \operatorname{diet}^{23}$. Although all our fly lines are in the $w^{1118}$ background, which is limited in tryptophan transport ${ }^{55}$, our innumerous control crosses (Figure 1) produced flies with varying expression levels of the transporter white (used as marker for the original transgenesis events that created the lines) with no apparent effect on viability on LN diet, strongly suggesting once more that the lethality is specifically caused by strong expression of AOX. These results are consistent with the idea that yeast extract alone does not have a properly balanced amino acid content to allow flies with strong expression of AOX to complete development. However, for total rescue of the AOX-driven pupal lethality, as seen with the addition of molasses to the LN $\operatorname{diet}^{23}$, a 
combination of proper levels of methionine or tryptophan, and of other nutrients might be required and is yet to be determined. Accomplishing this may not be simple, as even on SD diet, pupal viability of AOX-expressing flies seemed sensitive to our highest concentration of methionine supplementation (Figure 7A), which may also indicate the existence of an upper limit in the requirements of this amino acid for successful development these flies.

AOX-expressing larvae present metabolic markers of enhanced growth

In our larval metabolomics analysis, we also found elevated levels of 2-hydroxyglutarate (2-HG) caused independently by AOX and by LN diet (Figure 6). Along with succinate and fumarate (which was also elevated in AOX-expressing larvae, Figure 6), 2-HG is often considered an oncometabolite ${ }^{48}$, derived from $\alpha$-ketoglutarate by the action of Ldh in the cytoplasm $^{56,57}$. This reaction usually takes place when OXPHOS is somehow downregulated, and cytoplasmic NAD + must be regenerated by, among other enzymes, Ldh converting pyruvate into lactate. In fact, high levels of AOX expression in the larvae causes upregulation of $L d h$ transcripts (Supplemental Table S10) and an accumulation of lactate (Figure 6), which in turn may keep 2-HG levels high by acidifying the cellular environment and inhibiting its breakdown ${ }^{57,58}$. Interestingly, $2-\mathrm{HG}$, succinate and fumarate are inhibitors of $\alpha$-ketoglutaratedependent protein and DNA demethylases ${ }^{59}$. Their accumulation has independently been shown to cause epigenetic modifications ${ }^{58,60-62}$, and may also cause post-translational modifications in key enzymes, which we speculate may drive important changes in gene expression and/or metabolic flux that are necessary for the Drosophila larva to adapt to AOX function. The putative unbalanced levels of methionine/tryptophan (and likely other molecules) in the LN diet 
might actually impede these changes from occurring in AOX-expressing flies due to disturbed one-carbon metabolism and the lack of SAM.

Interestingly, in our previous report ${ }^{23}$, we showed that supplementation of the LN diet with a multivitamin complex containing folic acid did not altered the low viability rates of LNAOX pupae, perhaps because conversion of folic acid to 5-methyl-THF is dependent on a source of methyl groups, such as serine or glycine, which are supposedly not present at suitable levels in the $\mathrm{LN}$ diet for the proper development of AOX-expressing flies. Induction of the methionine cycle and production of SAM by methionine supplementation would bypass the need for other sources of methyl donors, but excess methionine can also compromise 5-methyl-THF synthesis by inhibiting the folate cycle enzyme methylene-THF reductase ${ }^{63,64}$, explaining the dosedependent partial rescue of the AOX-induced lethality seen in Figure 7A. Synthesis of 5-methylTHF using formate derived from tryptophan catabolism, on the other hand, would not cause such inhibition $^{64}$, perhaps explaining why LN supplementation with tryptophan alone in general increased pupal viability of LN-AOX flies better than supplementation with methionine alone, or methionine and tryptophan combined (Figure 7A).

Our metabolomics analysis also suggests that the AOX-expressing larvae have altered choline metabolism (Figure 6); oxidation of choline contributes to 5-methyl-THF synthesis and electrons to the mitochondrial electron transfer system ${ }^{64}$, providing further evidence for the importance of the one-carbon metabolism to the development of AOX-expressing larvae. Importantly, the changes in choline metabolites, which are apparent in the larval metabolomes, also suggest a link to the altered content of larval membrane lipids that we propose here. The choline metabolite phosphocholine, whose levels are significantly affected by the LN diet, AOX expression and the LN-AOX interaction (Figure 6), is both a precursor and a catabolic product of 
the phospholipid phosphatidylcholine ${ }^{39,65}$. Phosphoethanolamine, 3-PS and inositol, which are present in significantly altered levels in our metabolomics (Figure 6), are part of the polar head of the different forms of the phospholipids phosphatidylethanolamine, phosphatidylserine and phosphatidylinositol, respectively. These phospholipids, along with the diversity of sterol types that can support Drosophila development ${ }^{66}$, form the typical bilayer structure of cellular membranes and ultimately help control tissue integrity and function by regulating membrane integrity and selectivity ${ }^{39,67}$. Further experiments are warranted to show the links between the one-carbon and amino acid metabolisms, and phospholipid metabolism for the development of LN-AOX flies, but it is tempting to speculate that the putatively high requirement of AOXexpressing larvae for SAM in the limited nutrient availability condition of the LN diet might limit phospholipid synthesis, or drive their breakdown and the release of choline metabolites and 3-PS that could fuel the folate cycle.

The apparent dependence on the one-carbon metabolism for larval growth and completion of metamorphosis by the AOX-expressing flies is in fact intriguing. More interesting, perhaps, is the fact that many metabolic markers of increased tissue growth are present in AOXexpressing larvae, and many other aspects that are shared among proliferative tissues, such as tumors and the growing Drosophila larvae, appear more pronounced upon AOX expression. These include: 1) excessive formation and accumulation of lactate and 2-HG (Figure 6), associated with elevated $L d h$ levels (Supplemental Table S10); 2) accumulation and diversion of glycolytic and TCA cycle intermediates (Figure 6) to be supposedly used in the anabolism of amino acids, lipids, and nucleotides, among other molecules; 3 ) dependence on an extra source of essential amino acids, such as methionine and/or tryptophan (Figure 7); 4) abnormal choline and membrane phospholipid metabolism (Figure 6 and Supplemental Figure S5); 5) altered 
signaling pathways (Figure 5 and Supplemental Figure S8); among others ${ }^{50,53,65,68,69}$. In addition, as mentioned earlier, another important feature of proliferative tissues is the presence of mitochondria with a functional electron transfer system, so that $\mathrm{CoQH}_{2}$ can be reoxidized, allowing continuing or increased metabolic flux ${ }^{2}$. In fact, AOX expression is able to restore the overproliferative nature of cancer cells that are defective in CIII function and to promote tumors in mouse models ${ }^{2}$. It is therefore possible that the expression of AOX in fast growing tissues/systems that contain functional CIII/CIV complexes, such as our Drosophila larva model, may increase metabolic flux by increasing $\mathrm{CoQH}_{2}$ reoxidation.

In this hypothetical scenario, the reactions of the TCA cycle that promote mitochondrial $\mathrm{NAD}+$ regeneration for $\mathrm{CI}$ function would be accelerated, which in turn could also drive cataplerosis and induce larval growth, consistent with the changes in metabolism summarized above and, with the proposed increase in intestinal nutrient absorption and larval food consumption upon AOX expression (Figure 3). However, one would also expect increased larval biomass accumulation and/or faster development by AOX-expressing flies, at least when cultured on SD diet, but these have not been observed. On the contrary, we report here that AOX expression restricts larval weight gain even on SD diet, reaching almost $40 \%$ of biomass loss on LN diet, when compared to the controls cultured on SD diet (Figure 4). We then hypothesized that the problems with biomass accumulation might be caused specifically by the strong expression of AOX (as is the high pupal lethality rates), which in addition to increased cataplerosis, may also lead to mitochondrial decoupling due to the non-proton pumping nature of AOX, with concomitant production of excessive heat and/or compromise of mitochondrial ATP synthesis. Mitochondrial decoupling and heat production by AOX has been previously evidenced in cultured human cells ${ }^{7,70}$. We next tested if lower levels of AOX expression, which we showed 
not to cause lethality in combination with LN diet (Figure 1D), would have a positive impact on larval weight gain. In this case, mitochondrial uncoupling would not be as high (and ATP synthesis would perhaps be sufficiently compensated by the substrate-level phosphorylation reactions of glycolysis), but there would still be an increase in TCA cycle cataplerosis due to increased $\mathrm{CoQH}_{2}$ reoxidation, which could drive tissue growth. We selected the fly line $3 \mathrm{x} t u b A O X^{3}$, for which we have strong evidence of AOX-induced thermogenesis (and thus mitochondrial uncoupling $)^{22}$, and in which AOX is ubiquitously and constitutively expressed at least 5 fold less $^{24}$ than in the flies with strong expression of AOX used throughout this work. The AOX expression level in the $3 \mathrm{xtubAOX}$ flies is equivalent to what we defined as "intermediate" in Figure 1, which does not alter pupal viability, independently of diet. We observed a significant gain of $10-15 \%$ in larval size, and in wet and dry masses, independent of sex (Figure 8), when these flies were cultured on SD diet, which is in accordance with the idea that AOX expression induces growth in a dose-dependent fashion. These results, in combination with all other pieces of evidence we present here, suggest that tissue growth and biomass accumulation could be modulated by the xenotopic expression of AOX, the outcome of which may be determined by the balance between increased metabolic flux, increased mitochondrial uncoupling, and diet.

\section{Conclusions}

OXPHOS bypass using AOX stands as a potential strategy for future treatments of human mitochondrial and related diseases. To succeed in a safe way, the work with AOXexpressing animal models is crucial. We report here that the development of flies with strong ubiquitous expression of AOX is significantly impacted when cultured on our LN diet. In L3 larvae, transcriptomic changes are consistent with changes in intestinal morphology and in 
feeding behavior, suggesting that AOX induces an increased need for dietary nutrients. AOXexpressing larvae also present metabolic markers of enhanced growth, and higher storage of proteins and lipids. However, the LN diet drastically inhibits biomass accumulation in the form of lipids by the AOX-expressing larvae, causing on the pupae disturbed signaling processes, increased stress and starvation responses, and ultimately, developmental arrest. This arrest can be partially rescued by LN supplementation with methionine or tryptophan, indicating an important role for the one-carbon metabolism on the successful development of AOX-expressing flies. Neither lower levels of ubiquitous AOX expression nor high levels of tissue-specific expression lead to developmental arrest. In fact, lower (intermediate) ubiquitously-driven AOX-expressing larvae accumulate more biomass on SD diet, and have significantly better developmental outcome under cold stress $^{22}$, suggesting enhanced growth, yet energy dissipation as heat via mitochondrial uncoupling. Taken together, our work suggests that AOX may hormetically modulate growth by a balance between increased metabolic flux and increased mitochondrial uncoupling: the former promoting TCA cycle cataplerosis and anabolism, to a point at which the latter starts to affect ATP synthesis and/or produce too much metabolic heat, ultimately restricting growth (Figure 9).

Because the developmental arrest described here is dependent on the systemic impact caused by strong ubiquitous expression of AOX in combination with $\mathrm{LN}$ diet, it is in fact likely that AOX induces different metabolic changes depending on the functions of a particular tissue/organ. AOX expression might influence general larval growth by acting locally in each tissue/organ, but the problems in LN diet occur with the integration of these organs' functions. The musculature and the fat body, for example, are supposedly where the extra protein and fat of AOX-expressing larvae are respectively stored, and so, these are the tissues with the most 
elevated need for nutrients, and their mitochondria would participate significantly in the respective anabolic processes. However, to supply the extra nutrients needed, the nervous system and the intestines would need to be more active in inducing increased larval feeding behavior, in digesting the extra food, and in absorbing the extra nutrients. Mitochondria in these tissues would be primarily catabolic, instead, with high need to synthesize ATP. By applying wholelarva omics, we certainly missed most of the tissue-specific signals and metabolic changes promoted by AOX. Future studies will have to aim at understanding the tissue-specific metabolic disturbances caused by AOX in the growing Drosophila larva, and how they integrate to promote growth and biomass accumulation (or the lack thereof in LN diet).

The developing Drosophila larva has already been postulated as a model for our understanding of the metabolism of animal tissue growth, including that of cancer. It perhaps may even be a more realistic model than cancer cells cultured in vitro, since one can study how a complex living organism regulates metabolic flux intrinsically and in peripheral tissues, supporting the transitions through distinct developmental stages with varying metabolic demands $^{71}$. The idea that certain levels of AOX expression promote growth is worrisome from the point-of-view of a putative future treatment of human mitochondrial diseases, as it is still not known if the effects are merely hyperplastic, hypertrophic or also neoplastic. However, although highly speculative, the fact that the combination of strong expression of AOX and poor diet restricts growth may also indicate their potential applicability in cancer research. Better understanding the dose- and diet-dependent effects of AOX in tissue growth in the Drosophila larva will, therefore, be extremely relevant.

\section{Materials and Methods}




\section{Fly stock and diets}

The D. melanogaster recipient line $w^{1118}$, the driver lines daughterless-GAL4 (daGAL4), embryonic lethal abnormal visionC155-GAL4 (elavGAL4), myosin heavy chain-GAL4 (mhcGAL4) and r-tetramer-GAL4 (r4GAL4), and the GAL4-dependent UAS-GFPStinger line, were obtained from stock centers. The driver line drumstick-GAL4 (drmGAL4) was a gift from Dr. Lucas Anhezini's lab. Previously described transgenic lines carrying tubAOX, UAS-AOX constructs or the $U A S$ promoter only ("empty" pUASTattB plasmid) were used: $3 \mathrm{x} t u b A O X$ (inserted into chromosomes $\mathrm{X}, 2$ and 3$)^{3}, U A S-A O X^{F 6}$ (inserted into chromosome 2$)^{10}, U A S$ $A O X^{8.1}$ (chromosome 2), UAS-AOX $X^{7.1}$ (chromosome 3), UAS-mutAOX ${ }^{2 n d}$ (chromosome 2), UASmutAOX ${ }^{3 r d}$ (chromosome 3) and UAS-empty ${ }^{2 n d}$ (chromosome 2) ${ }^{16}$. The double transgenic lines $U A S-A O X^{8.1} ; U A S-A O X^{7.1}$ and $U A S-m u t A O X^{2 n d} ; U A S-m u t A O X^{3 r d}$ were created by standard genetic crosses using lines carrying traditional balancer chromosomes. All fly lines were backcrossed with the recipient line $w^{1118}$ six-to-ten generations prior to the experiments. The fly lines were maintained on SD diet ${ }^{10}$, with a $12 / 12 \mathrm{~h}$ of light/dark photoperiod cycle at 18 or $25^{\circ} \mathrm{C}$. The LN diet consisted of $3.5 \%$ yeast extract, $1 \%$ agar and antimicrobials $(0.1 \%$ niapigin and $0.5 \%$ propionic acid). For the experiments shown in Figure 7, both SD and LN diets were supplemented with the amino acids methionine, tryptophan, proline and glutamate at the concentrations indicated in the figure legend.

\section{Developmental assays}

The UAS-AOX,UAS-GFPStinger and UAS-empty lines were crossed to either a GAL4 driver line to induce transgene expression, or to the recipient line $w^{1118}$ (uninduced hemizygous controls), as indicated in the figure legends. Based on immunoblot analyses (Figure 1B) and 
previously published work ${ }^{16}$, the crosses between $U A S-A O X^{F 6}$ and the $G A L 4$ drivers generated flies with high expression levels of AOX, whereas the same crosses using $U A S-A O X^{8.1}$ or $U A S$ $A O X^{7.1}$ produced low levels of AOX. The double transgenic lines $U A S-A O X^{8.1} ; U A S-A O X^{7.1}$ and $U A S$-mutAOX ${ }^{2 n d} ; U A S$-mutAOX ${ }^{3 r d}$, upon crossing with GAL4 drivers, and the triple transgenic line $3 \mathrm{x} t u b A O X$ (constitutive expression) generated flies with intermediate expression levels of AOX. Ten-fifteen female virgins and 5-7 males were pre-mated on SD diet during 24-48h at 25 ${ }^{\circ} \mathrm{C}$ with a 12/12h light/dark photoperiod cycle, flipped into vials containing SD or LN diets, and allowed to lay eggs until the limiting rate of 60-80 eggs per vial was reached. The eggs were allowed to develop in the same vials, from which pupae and adults were recorded. Pupal viability was calculated as the ratio between eclosed adults and total number of pupae, averaged among 23 biological replicates, each carrying 5-8 vials (technical replicates).

\section{Measurements of body mass, intestinal length, food consumption, and fat and protein content}

For larval body mass measurements, batches of ten randomly selected, wandering L3 larvae (100-115 h post-egg laying in SD diet; 125-140 h in LN diet) were collected, briefly rinsed in deionized water, carefully dried in Kimwipes ${ }^{\circledR}$ sheets, placed in a pre-weighted $1.5 \mathrm{ml}$ microcentrifuge tube, and weighed using the precision balance ATX224 (Shimadzu Scientific Instruments) to obtain the wet mass. The tubes were then frozen at $-20^{\circ} \mathrm{C}$ for up to 14 days, and placed in a dry bath at $65^{\circ} \mathrm{C}$ with the lids open until the larval weight dropped to constant values $(\sim 2 \mathrm{~h})$, allowing us to record the dry mass weight. Next, the samples were incubated with $1 \mathrm{~mL}$ ethyl ether for $24 \mathrm{~h}$ at room temperature, and the procedure was further repeated three times, when the samples were then dried as described above, allowing us to obtain the lean mass weight. Individual larval masses were calculated by dividing the total weight per tube by 10 , and 
the final values were obtained by averaging the individual larval masses of $\sim 40$ tubes, obtained in three independent experiments. Measurements of total fat content were obtained by subtracting lean mass weight from dry mass weight.

For estimates of total protein content, batches of 20 randomly selected, wandering L3 larvae (100-115 h post-egg laying in SD diet; 125-140 h in LN diet) were collected, briefly rinsed in deionized water, carefully dried in Kimwipes ${ }^{\circledR}$ sheets, and homogenized on ice with 1 $\mathrm{mL}$ isolation buffer (250 mM sucrose, $5 \mathrm{mM}$ Tris-HCl pH 7.5, 2 mM EGTA, pH 7.4) using a handheld glass homogenizer with 3-5 strokes. Total protein quantitation was obtained by the Bradford method. Individual larval protein content was calculated by dividing the total protein per tube by 20 , and the final values were obtained by averaging 4-6 independent experiments.

For measurements of intestinal length, randomly selected, wandering L3 larvae (100-115 $\mathrm{h}$ post-egg laying in SD diet; 125-140 $\mathrm{h}$ in LN diet) of each sex were collected, briefly rinsed in deionized water, and submerged in $\sim 80^{\circ} \mathrm{C}$ water for $\sim 2 \mathrm{~s}$ for immobilization. The individuals were immediately photographed and then transferred to a dish containing $1 \mathrm{X}$ PBS (8 mM $\mathrm{Na}_{2} \mathrm{HPO}_{4}, 2 \mathrm{mM} \mathrm{KH} \mathrm{PO}_{4}, 150 \mathrm{mM} \mathrm{NaCl}, 30 \mathrm{mM} \mathrm{KCl}, \mathrm{pH}$ 7.4). The intestines were immediately dissected and photographed. The images were analyzed and quantitated using the software Image J v1.53e. The ratio between intestinal length and larval area was averaged from measurements of $\sim 15$ individuals of each sex, in three independent experiments.

Measurements of larval food consumption were adapted from ${ }^{72}$. Briefly, randomly selected, early L3 larvae (75-85 h post-egg laying in SD diet; 95-105 h in LN diet) were collected, rinsed in deionized water, and placed on a dish without food for $20 \mathrm{~min}$ at $25^{\circ} \mathrm{C}$. The individuals were then transferred to a dish with $6 \mathrm{ml}$ of food containing $2 \mathrm{~g}$ yeast extract and 15 mg Brilliant blue R dye, and allowed to feed for 10 min, after which they were processed and 
their intestines were dissected and photographed, as described above. The images were analyzed and the blue signal intensity was quantitated using the software ImageJ v1.53e. The ratio between the blue signal intensity and intestinal length was averaged from measurements of seven-ten individuals, in two-three independent experiments.

\section{Immunoblotting}

Mitochondrial protein extracts were prepared on ice by homogenization of 20 randomly selected, whole adult males (1-3 days after eclosion) of the indicated genotype on isolation buffer (250 mM sucrose, 5 mM Tris-HCl pH 7.5, 2 mM EGTA, pH 7.4), followed by centrifugation at $200 \times \mathrm{g}$ for $1 \mathrm{~min}$ at $4^{\circ} \mathrm{C}$. The supernatant was removed and centrifuged again at $200 \times \mathrm{g}$ for $3 \mathrm{~min}$ at $4^{\circ} \mathrm{C}$. The recovered supernatant was further centrifuged at $9,000 \times \mathrm{g}$ for 10 min at $4^{\circ} \mathrm{C}$, and the mitochondrial pellet was resuspended in $50 \mu \mathrm{L}$ isolation buffer. The protein quantification was performed by the Bradford method, and the samples were stored at $-80^{\circ} \mathrm{C}$ until use. Forty $\mu \mathrm{g}$ of mitochondrial protein extract were mixed with Laemmli buffer (2\% SDS, $10 \%$ glycerol, $5 \%$ 2- mercaptoethanol, $0.02 \%$ bromophenol blue and $62.5 \mathrm{mM}$ Tris- $\mathrm{HCl}, \mathrm{pH}$ 6.8), denatured at $95^{\circ} \mathrm{C}$ for $3 \mathrm{~min}$, and resolved on $12 \%$ SDS-polyacrylamide gels for $\sim 3.5 \mathrm{~h}$ at $80 \mathrm{~V}$, alongside the Bio-Rad Precision Plus Protein Standards marker. The proteins were transferred at $550 \mathrm{~mA}$ for $7 \mathrm{~min}$ to nitrocellulose membranes using the semi-dry transfer system MSD10 (Major Science) at room temperature. The membranes were blocked in 5\% milk/PBST solution ( $8 \mathrm{mM} \mathrm{Na}_{2} \mathrm{HPO}_{4}, 2 \mathrm{mM} \mathrm{KH} \mathrm{PO}_{4}, 150 \mathrm{mM} \mathrm{NaCl}, 30 \mathrm{mM} \mathrm{KCl}, 0.05 \%$ Tween 20, 5\% dried nonfat milk, $\mathrm{pH} 7.4$ ) for at least $2 \mathrm{~h}$, and incubated with a 1\% milk/PBST solution containing the primary antibodies anti-AOX (rabbit polyclonal, 1:20,000) and anti-ATP5A (mouse monoclonal, 1:10,000) for $3 \mathrm{~h}$ at room temperature under constant shaking. After 
washing $3 \mathrm{X}$ with PBST, 40 min each, the membranes were incubated with a $1 \%$ milk/PBST solution containing the secondary HRP-conjugated goat anti-rabbit $(1: 10,000$, Bio-Rad) and antimouse $\left(1: 10,000\right.$, Bio-Rad) antibodies overnight at $4^{\circ} \mathrm{C}$, and washed as previously. Chemiluminescence signals were detected on a ChemiDoc Imaging System (Bio-Rad), after the membranes were incubated with the luminol substrate detection system Immun-Star HRP (Bio$\mathrm{Rad})$.

\section{$R N A$ extraction, sequencing and data analyses}

Ten randomly selected, wandering L3 larvae (100-115 hrs post-egg laying in SD diet; 125-140 hrs in LN diet) and 10 pre-pharate pupae (65-80 hrs post-pupariation) per biological replica (3 in total) were collected, briefly rinsed in ultrapure water to remove dietary residues, and immediately processed. Total RNA was extracted using the RNeasy® Tissue kit (QIAGEN), according to manufacturer instructions, eluted in nuclease-free water, and analyzed using the Qubit ${ }^{\circ}$ RNA HS Assay kit (Invitrogen) and the RNA 6000 Nano LabChip kit/Bioanalyzer 2100 (Agilent Technologies). One $\mu \mathrm{g}$ of RNA with high integrity number (RINe $>8.9$ ) were used for RNA sequencing library preparation using the TruSeq Stranded mRNA Library Prep Kit (Illumina), according to the manufacturer`s protocol. The libraries were quantified using the Promega QuantiFluor dsDNA System on a Quantus Fluorometer (Promega), and analyzed using the High Sensitivity D1000 Screen Tape on an Agilent 2200 TapeStation instrument. The libraries were normalized, pooled, and subjected to cluster generation, and paired-end sequencing was performed for $2 \times 150$ cycles on a HiSeq4000 instrument (Illumina), according to the manufacturer's instructions, by the company Omega Bioservices (Norcross, GA, USA). The 
unprocessed data was deposited in the NCBI Sequence Read Archive under the accession number BioProject PRJNA763657.

The sequencing reads were processed with Trimmomatic ${ }^{73}$ and assessed by FastQC ${ }^{74}$ for processing quality. The alignment of the reads was performed with HISAT2 ${ }^{75}$ using the $D$. melanogaster reference genome r6.30, available on FlyBase (flybase.org). The quantitation of mapped reads was performed by $\mathrm{HTSeq}^{76}$; reads with more than 5 counts in at least 3 libraries were considered for the analyses. The statistical analysis was performed using the edgeR package $^{77}$ in the multifactorial analysis mode. The factors considered were diet, AOX expression and the interaction between diet and AOX. Genes differentially expressed were determined as $p$ $<0.05$. Gene ontology analysis was performed considering each factor using DAVID v6.8 ${ }^{78}$. Unsupervised clustering was performed by the Cluster3.0 software, and the heatmaps were created through JAVA TreeView v1.1.6r4.

\section{Metabolite extraction and mass spectrometry analyses}

Ten randomly selected, wandering L3 larvae (100-115 hrs post-egg laying in SD diet; 125-140 hrs in LN diet) per biological replica (5 in total) were collected, briefly rinsed in ultrapure water, and homogenized in $1 \mathrm{ml}$ cold $\left(-80^{\circ} \mathrm{C}\right) 80 \%$ methanol on dry ice using a probe homogenizer. Samples were clarified by centrifugation for $5 \mathrm{~min}$ at $16,000 \mathrm{x} g_{\max }$ at $4{ }^{\circ} \mathrm{C}$ and the supernatants were transferred to new Eppendorf tubes. The remaining pellets were resuspended in RIPA lysis buffer and total protein content was determined by the BCA method. A volume of the supernatant equivalent to $5 \mu \mathrm{g}$ of protein was transferred to a glass vial, dried using an EZ-2 Elite evaporator (SP Scientific), and stored at $-80^{\circ} \mathrm{C}$ until analysis by LC-MS. The samples were analyzed using an UltiMate 3000RSLC HPLC (Thermo Scientific) coupled to a Q Exactive mass 
spectrometer (Thermo Scientific). After resuspension in 50\% acetonitrile, $1 / 10$ th of the samples

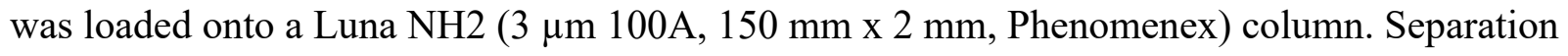
was achieved with $5 \mathrm{mM} \mathrm{NH}_{4} \mathrm{OAc}$, pH 9.9 (mobile phase A) and $\mathrm{ACN}$ (mobile phase B) at a flow rate of $200 \mu \mathrm{l} / \mathrm{min}$ and a linear gradient from $15 \%$ to $90 \% \mathrm{~A}$ over $18 \mathrm{~min}$. This was followed by an isocratic step at 90\% A for 9 min and re-equilibration to the initial 15\% A for 7 min. The Q Exactive was run with polarity switching $(+3.50 \mathrm{kV} /-3.50 \mathrm{kV})$ in full scan mode with an $\mathrm{m} / \mathrm{z}$ range of 65-975. Metabolites were identified with TraceFinder 4.1 (Thermo Fisher Scientific) using accurate mass measurements $(\leq 3 \mathrm{ppm})$ and expected retention times previously established with pure standards. Quantities were determined by peak area integration.

\section{Acknowledgements}

We would like to thank Dr. Lucas Anhezini for providing the drmGAL4 line, and Dr. Richard Hensh for help with the statistical analyses. A.F.C., M.M.C., G.S.G., M.F.O. and A.A.M. were supported by fellowships from the Coornadoria de Aperfeiçoamento de Pessoal de Nível Superior (CAPES, grant numbers 88882.434433/2019-01 and 88882.434441/2019-01), Conselho Nacional de Desenvolvimento Científico e Tecnológico (CNPq, grant number 141001/2019-4), and Fundação de Amparo à Pesquisa do Estado de São Paulo (FAPESP, grant numbers 1207/13743-2, 2020/07797-5 and 2017/17645-5). M.T.O. would like to acknowledge funding from FAPESP (grant numbers 2014/02253-6 and 2017/04372-0), CNPq (grant numbers 424562/2018-9 and 306974/2017-7) and the European Union (Marie Curie International Incoming Fellowship GA328988). E.D. acknowledges funding from AFM-Telethon (research grant number 17424) and the Academy of Finland (research fellowship number 130172). 


\section{References}

1. Jacobs, H. T., George, J. \& Kemppainen, E. Regulation of growth in Drosophila melanogaster: the roles of mitochondrial metabolism. J. Biochem. 167, 267-277 (2020).

2. Martínez-Reyes, I. et al. Mitochondrial ubiquinol oxidation is necessary for tumour growth. Nature 585, 288-292 (2020).

3. Kemppainen, K. K. et al. Expression of alternative oxidase in Drosophila ameliorates diverse phenotypes due to cytochrome oxidase deficiency. Hum. Mol. Genet. 23, 20782093 (2014).

4. Gorman, G. S. et al. Mitochondrial diseases. Nat. Rev. Dis. Prim. 2, 16080 (2016).

5. McDonald, A. E., Vanlerberghe, G. C. \& Staples, J. F. Alternative oxidase in animals: unique characteristics and taxonomic distribution. J. Exp. Biol. 212, 2627-2634 (2009).

6. Sanz, A., Fernández-Ayala, D. J. M., Stefanatos, R. K. \& Jacobs, H. T. Mitochondrial ROS production correlates with, but does not directly regulate lifespan in drosophila. Aging (Albany. NY). 2, 200-223 (2010).

7. Cannino, G. et al. Glucose Modulates Respiratory Complex I Activity in Response to Acute Mitochondrial Dysfunction. J. Biol. Chem. 287, 38729-38740 (2012).

8. Saha, B., Borovskii, G. \& Panda, S. K. Alternative oxidase and plant stress tolerance. Plant Signal. Behav. 11, e1256530 (2016).

9. Hakkaart, G. A. J., Dassa, E. P., Jacobs, H. T. \& Rustin, P. Allotopic expression of a mitochondrial alternative oxidase confers cyanide resistance to human cell respiration. EMBO Rep. 7, 341-345 (2006).

10. Fernandez-Ayala, D. J. M. et al. Expression of the Ciona intestinalis Alternative Oxidase (AOX) in Drosophila Complements Defects in Mitochondrial Oxidative Phosphorylation. Cell Metab. 9, 449-460 (2009).

11. El-Khoury, R. et al. Alternative Oxidase Expression in the Mouse Enables Bypassing Cytochrome c Oxidase Blockade and Limits Mitochondrial ROS Overproduction. PLoS Genet. 9, e1003182 (2013).

12. El-Khoury, R. et al. Expression of the alternative oxidase mitigates beta-amyloid production and toxicity in model systems. Free Radic. Biol. Med. 96, 57-66 (2016).

13. Szibor, M. et al. Broad AOX expression in a genetically tractable mouse model does not disturb normal physiology. Dis. Model. Mech. 10, 163-171 (2017).

14. Giordano, L. et al. Alternative Oxidase Attenuates Cigarette Smoke-induced Lung Dysfunction and Tissue Damage. Am. J. Respir. Cell Mol. Biol. 60, 515-522 (2019). 
15. Dassa, E. P. et al. Expression of the alternative oxidase complements cytochrome c oxidase deficiency in human cells. EMBO Mol. Med. 1, 30-36 (2009).

16. Andjelković, A. et al. Diiron centre mutations in Ciona intestinalis alternative oxidase abolish enzymatic activity and prevent rescue of cytochrome oxidase deficiency in flies. Sci. Rep. 5, (2015).

17. Rajendran, J. et al. Alternative oxidase-mediated respiration prevents lethal mitochondrial cardiomyopathy. EMBO Mol. Med. 11, e9456 (2019).

18. Rustin, P. \& Jacobs, H. T. Respiratory chain alternative enzymes as tools to better understand and counteract respiratory chain deficiencies in human cells and animals. Physiol. Plant. 137, 362-370 (2009).

19. Giordano, L. et al. Alternative oxidase encoded by sequence-optimized and chemicallymodified RNA transfected into mammalian cells is catalytically active. Gene Ther. (2021) doi:10.1038/s41434-021-00235-z.

20. McDonald, A. E. \& Gospodaryov, D. V. Alternative NAD(P)H dehydrogenase and alternative oxidase: Proposed physiological roles in animals. Mitochondrion 45, 7-17 (2019).

21. Dogan, S. A. et al. Perturbed Redox Signaling Exacerbates a Mitochondrial Myopathy. Cell Metab. 28, 764-775.e5 (2018).

22. Saari, S. et al. Alternative respiratory chain enzymes: Therapeutic potential and possible pitfalls. Biochim. Biophys. Acta - Mol. Basis Dis. 1865, 854-866 (2019).

23. Saari, S. et al. Alternative oxidase confers nutritional limitation on Drosophila development. J. Exp. Zool. Part A Ecol. Integr. Physiol. 331, 341-356 (2019).

24. Saari, S., Andjelković, A., Garcia, G. S., Jacobs, H. T. \& Oliveira, M. T. Expression of Ciona intestinalis AOX causes male reproductive defects in Drosophila melanogaster. BMC Dev. Biol. 17, 9 (2017).

25. Garcia, G. S., Othonicar, M. F., Oliveira, M. T. \& Couto-Lima, C. A. An Affordable and Efficient 'Homemade' Platform for Drosophila Behavioral Studies, and an Accompanying Protocol for Larval Mitochondrial Respirometry. J. Vis. Exp. in press.

26. Rodrigues, A. P. C., Camargo, A. F., Andjelković, A., Jacobs, H. T. \& Oliveira, M. T. Developmental arrest in Drosophila melanogaster caused by mitochondrial DNA replication defects cannot be rescued by the alternative oxidase. Sci. Rep. 8, 10882 (2018).

27. Camargo, A. F. et al. Xenotopic expression of alternative electron transport enzymes in animal mitochondria and their impact in health and disease. Cell Biol. Int. 42, 664-669 (2018).

28. Kemppainen, K. K., Kemppainen, E. \& Jacobs, H. T. The Alternative Oxidase AOX Does 
Not Rescue the Phenotype of tko25t Mutant Flies. G3 Genes $\mid$ Genomes $\mid$ Genetics 4, 20132021 (2014).

29. Andjelković, A. et al. Expression of the Alternative Oxidase Influences Jun N-Terminal Kinase Signaling and Cell Migration. Mol. Cell. Biol. 38, e00110-18 (2018).

30. Graveley, B. R. et al. The developmental transcriptome of Drosophila melanogaster. Nature 471, 473-479 (2011).

31. Daines, B. et al. The Drosophila melanogaster transcriptome by paired-end RNA sequencing. Genome Res. 21, 315-324 (2011).

32. Tennessen, J. M., Baker, K. D., Lam, G., Evans, J. \& Thummel, C. S. The Drosophila Estrogen-Related Receptor Directs a Metabolic Switch that Supports Developmental Growth. Cell Metab. 13, 139-148 (2011).

33. Tennessen, J. M. \& Thummel, C. S. Coordinating Growth and Maturation - Insights from Drosophila. Curr. Biol. 21, R750-R757 (2011).

34. Martin-Blanco, E., Pastor-Pareja, J. C. \& Garcia-Bellido, A. JNK and decapentaplegic signaling control adhesiveness and cytoskeleton dynamics during thorax closure in Drosophila. Proc. Natl. Acad. Sci. 97, 7888-7893 (2000).

35. Brown, J. B. et al. Diversity and dynamics of the Drosophila transcriptome. Nature 512, 393-399 (2014).

36. No Title. https://lemaitrelab.epfl.ch/resources.

37. Merzendorfer, H. \& Zimoch, L. Chitin metabolism in insects: structure, function and regulation of chitin synthases and chitinases. J. Exp. Biol. 206, 4393-4412 (2003).

38. Miguel-Aliaga, I., Jasper, H. \& Lemaitre, B. Anatomy and Physiology of the Digestive Tract of Drosophila melanogaster. Genetics 210, 357-396 (2018).

39. Carvalho, M. et al. Effects of diet and development on the Drosophila lipidome. Mol. Syst. Biol. 8, 600 (2012).

40. Honegger, B. et al. Imp-L2, a putative homolog of vertebrate IGF-binding protein 7, counteracts insulin signaling in Drosophila and is essential for starvation resistance. $J$. Biol. 7, 10 (2008).

41. Garelli, A., Gontijo, A. M., Miguela, V., Caparros, E. \& Dominguez, M. Imaginal Discs Secrete Insulin-Like Peptide 8 to Mediate Plasticity of Growth and Maturation. Science (80-. ). 336, 579-582 (2012).

42. Colombani, J., Andersen, D. S. \& Leopold, P. Secreted Peptide Dilp8 Coordinates Drosophila Tissue Growth with Developmental Timing. Science (80-. ). 336, 582-585 (2012). 
43. Ray, M. \& Lakhotia, S. C. Activated Ras/JNK driven Dilp8 in imaginal discs adversely affects organismal homeostasis during early pupal stage in Drosophila, a new checkpoint for development. Dev. Dyn. 248, 1211-1231 (2019).

44. Wu, M., Pastor-Pareja, J. C. \& Xu, T. Interaction between RasV12 and scribbled clones induces tumour growth and invasion. Nature 463, 545-548 (2010).

45. von Frieling, J. et al. A high-fat diet induces a microbiota-dependent increase in stem cell activity in the Drosophila intestine. PLOS Genet. 16, e1008789 (2020).

46. Huang, K. et al. Impaired peroxisomal import in Drosophila oenocytes causes cardiac dysfunction by inducing upd3 as a peroxikine. Nat. Commun. 11, 2943 (2020).

47. Wisidagama, D. R. \& Thummel, C. S. Regulation of Drosophila Intestinal Stem Cell Proliferation by Enterocyte Mitochondrial Pyruvate Metabolism. G3 Genes $\mid$ Genomes|Genetics 9, 3623-3630 (2019).

48. Tretter, L., Patocs, A. \& Chinopoulos, C. Succinate, an intermediate in metabolism, signal transduction, ROS, hypoxia, and tumorigenesis. Biochim. Biophys. Acta - Bioenerg. 1857, 1086-1101 (2016).

49. Kumar, A. et al. The metabolism and significance of homocysteine in nutrition and health. Nutr. Metab. (Lond). 14, 78 (2017).

50. Kaiser, P. Methionine Dependence of Cancer. Biomolecules 10, 568 (2020).

51. Yang, M. \& Vousden, K. H. Serine and one-carbon metabolism in cancer. Nat. Rev. Cancer 16, 650-662 (2016).

52. Locasale, J. W. Serine, glycine and one-carbon units: cancer metabolism in full circle. Nat. Rev. Cancer 13, 572-583 (2013).

53. Sanderson, S. M., Gao, X., Dai, Z. \& Locasale, J. W. Methionine metabolism in health and cancer: a nexus of diet and precision medicine. Nat. Rev. Cancer 19, 625-637 (2019).

54. Yalçın, S., Erol, H., Özsoy, B., Onbaşılar, I. \& Yalçın, S. Effects of the usage of dried brewing yeast in the diets on the performance, egg traits and blood parameters in quails. Animal 2, 1780-1785 (2008).

55. Sullivan, D. T. \& Sullivan, M. C. Transport defects as the physiological basis for eye color mutants of Drosophila melanogaster. Biochem. Genet. 13, 603-613 (1975).

56. Teng, X., Emmett, M. J., Lazar, M. A., Goldberg, E. \& Rabinowitz, J. D. Lactate Dehydrogenase C Produces S-2-Hydroxyglutarate in Mouse Testis. ACS Chem. Biol. 11, 2420-2427 (2016).

57. Li, H. et al. Drosophila larvae synthesize the putative oncometabolite L-2hydroxyglutarate during normal developmental growth. Proc. Natl. Acad. Sci. 114, 1353- 


\section{8 (2017).}

58. Li, H., Hurlburt, A. J. \& Tennessen, J. M. A Drosophila model of combined D-2- and L-2hydroxyglutaric aciduria reveals a mechanism linking mitochondrial citrate export with oncometabolite accumulation. Dis. Model. Mech. 11, dmm035337 (2018).

59. Martínez-Reyes, I. \& Chandel, N. S. Mitochondrial TCA cycle metabolites control physiology and disease. Nat. Commun. 11, 102 (2020).

60. Xu, W. et al. Oncometabolite 2-Hydroxyglutarate Is a Competitive Inhibitor of $\alpha$ Ketoglutarate-Dependent Dioxygenases. Cancer Cell 19, 17-30 (2011).

61. Lu, C. et al. IDH mutation impairs histone demethylation and results in a block to cell differentiation. Nature 483, 474-478 (2012).

62. Sciacovelli, M. et al. Fumarate is an epigenetic modifier that elicits epithelial-tomesenchymal transition. Nature 537, 544-547 (2016).

63. WAYDHAS, C., WEIGL, K. \& SIES, H. The Disposition of Formaldehyde and Formate Arising from Drug N-Demethylations Dependent on Cytochrome P-450 in Hepatocytes and in Perfused Rat Liver. Eur. J. Biochem. 89, 143-150 (1978).

64. Brosnan, M. E., MacMillan, L., Stevens, J. R. \& Brosnan, J. T. Division of labour: how does folate metabolism partition between one-carbon metabolism and amino acid oxidation? Biochem. J. 472, 135-146 (2015).

65. Glunde, K., Bhujwalla, Z. M. \& Ronen, S. M. Choline metabolism in malignant transformation. Nat. Rev. Cancer 11, 835-848 (2011).

66. Carvalho, M. et al. Survival strategies of a sterol auxotroph. Development 137, 3675-3685 (2010).

67. Mountford, C. E. \& Wright, L. C. Organization of lipids in the plasma membranes of malignant and stimulated cells: a new model. Trends Biochem. Sci. 13, 172-177 (1988).

68. Vaupel, P. \& Multhoff, G. Revisiting the Warburg effect: historical dogma versus current understanding. J. Physiol. 599, 1745-1757 (2021).

69. Platten, M., Nollen, E. A. A., Röhrig, U. F., Fallarino, F. \& Opitz, C. A. Tryptophan metabolism as a common therapeutic target in cancer, neurodegeneration and beyond. Nat. Rev. Drug Discov. 18, 379-401 (2019).

70. Chrétien, D. et al. Mitochondria are physiologically maintained at close to $50{ }^{\circ} \mathrm{C} . P L O S$ Biol. 16, e2003992 (2018).

71. Drummond-Barbosa, D. \& Tennessen, J. M. Reclaiming Warburg: using developmental biology to gain insight into human metabolic diseases. Development 147, dev189340 (2020). 
72. Kaun, K. R. et al. Natural variation in food acquisition mediated via a Drosophila cGMPdependent protein kinase. J. Exp. Biol. 210, 3547-3558 (2007).

73. Bolger, A. M., Lohse, M. \& Usadel, B. Trimmomatic: a flexible trimmer for Illumina sequence data. Bioinformatics 30, 2114-2120 (2014).

74. Andrews, S. FastQC: a quality control tool for high throughput sequence data. (2010).

75. Kim, D., Langmead, B. \& Salzberg, S. L. HISAT: a fast spliced aligner with low memory requirements. Nat. Methods 12, 357-360 (2015).

76. Anders, S., Pyl, P. T. \& Huber, W. HTSeq--a Python framework to work with highthroughput sequencing data. Bioinformatics 31, 166-169 (2015).

77. Robinson, M. D., McCarthy, D. J. \& Smyth, G. K. edgeR: a Bioconductor package for differential expression analysis of digital gene expression data. Bioinformatics 26, 139140 (2010).

78. Jiao, X. et al. DAVID-WS: a stateful web service to facilitate gene/protein list analysis. Bioinformatics 28, 1805-1806 (2012).

79. Grandison, R. C., Piper, M. D. W. \& Partridge, L. Amino-acid imbalance explains extension of lifespan by dietary restriction in Drosophila. Nature 462, 1061-1064 (2009). 


\section{Figure Legends}

Figure 1. Pupal lethality is achieved via interaction between low nutrient diet and strong ubiquitous expression of AOX. A, Pupal viability of individuals with ubiquitous induction (ind) or not (unind) of AOX expression at the indicated levels. AOX expression was accomplished by crossing the homozygous $d a G A L 4$ line with homozygous $U A S$ - $A O X^{F 6}{ }^{10}$ for strong levels, $U A S$ $A O X^{8.1} ; U A S-A O X^{7.1}$ for intermediate levels, $U A S-A O X^{8.1}$ and $U A S-A O X^{7.116}$ for weak 1 and 2 levels, and $U A S$-mutAOX ${ }^{2 n d}$; UAS-mutAOX ${ }^{3 r d}$ for the catalytically-inactive mutant form of $\mathrm{AOX}^{16}$. Uninduced controls were progeny of the same $U A S$ lines and $w^{1118}$. B, Immunoblot showing levels in whole body samples of a mitochondrial protein marker, ATP5A, and AOX when its expression was ubiquitously induced at strong (S), intermediate (I) or weak (W1, W2) levels at the indicated diet. The same lines described in A were used here to reach the indicated AOX levels. M represents AOX expression levels in the mutant AOX line. Note that this mutant protein in this condition is not as stable as the wild type AOX: first, protein levels were expected to be similar between samples of the mutant AOX and the intermediate wild type AOX lines, but the mutant AOX protein is decreased; and second, an additional, smaller band likely representing a proteolytic product is observed in flies cultured on the low nutrient diet. C, AOX transcript levels extracted from the RNA-seq data of whole body samples of flies with strong ubiquitous expression of AOX (progeny of $U A S-A O X^{F 6}$ and $d a G A L 4$ ) and uninduced controls (progeny of $U A S-A O X^{F 6}$ and $\left.w^{1118}\right)$ at the indicated developmental stages, cultured at the indicated diet. The data was normalized by the read counts of AOX transcripts in the uninduced controls cultured in standard diet, which was arbitrarily set to 1.0. D, Pupal viability of control flies, and flies with tissue-specific induction or not (unind hemiz controls) of strong expression of AOX in muscles, 
neurons or fat body, when cultured at the indicated diet. bkg, genetic background; homoz, homozygous; hemiz, hemizygous; unind, uninduced. The homoz driver and unind homoz controls represent, respectively, the GAL4 driver and $U A S$ lines in homozygosity. The hemiz driver and unind hemiz controls represent, respectively, flies with a single copy of the transgenic GAL4 and $U A S$ constructs in hemizygosity, progeny of the cross between the homozygous lines with $w^{1118}$. Tissue-specific induction was accomplished by crossing homozygous GAL4 with $U A S$ lines, resulting in individuals with a single copy of each construct in hemizygosity. GFP, $U A S$-StingerGFP; strong AOX, UAS-AOX ${ }^{F 6}{ }^{10}$. The data in A, C and D represent average of two or three experiments; error bars represent standard deviations. a and b represent significantly different statistical classes, according to a one-way ANOVA, followed by the Tukey post-hoc test.

Figure 2. Transcripts involved with proteolysis and chitin metabolism are commonly affected by the low nutrient (LN) diet and/or AOX expression in larvae. The heatmaps show the transcripts altered by (A) LN diet, (B) strong ubiquitous AOX expression, and the (C) LN-AOX interaction. The columns LN, AOX and LN-AOX show respectively color representations of the $\log _{2}$ (fold change) values of the indicated transcript in control larvae cultured in LN diet, AOX-expressing larvae cultured in standard (SD) diet, and AOX-expressing larvae culture in LN diet, each in a pairwise comparison with control larvae cultured in SD diet. The indicated genes were selected as significantly differentially expressed $(\mathrm{p}<0.05)$ according to a multifactorial analysis performed using RNA-seq data and the software edgeR ${ }^{77}$. DAVID functional annotation tool ${ }^{78}$ was used to generate the indicated gene clusters (see Supplemental Figures S1-S3 for the remaining clusters assigned for each condition, and Material and Methods for experimental 
details). Control larvae, progeny of $U A S-A O X^{F 6}$ and $w^{1118}$; AOX-expressing larvae, progeny of $U A S-A O X^{F 6}$ and $d a G A L 4$.

Figure 3. Impact of AOX expression on the larval intestines and food consumption. A, The intestines were dissected from larvae with strong ubiquitous expression of $\mathrm{AOX}(d a>$ strong AOX, progeny of $U A S-A O X^{F 6}$ and $d a G A L 4$ ) and the uninduced hemizygous controls (progeny of $U A S-A O X^{F 6}$ and $w^{1118}$ ) cultured at the indicated diet, and tissues were processed as described in the Materials and Methods. The data was normalized by the relative intestinal length of the uninduced controls cultured in standard diet, which was arbitrarily set to 1.0 . a and b represent significantly different statistical classes, according to a two-way ANOVA, followed by the Tukey post-hoc test. B, Pupal viability of flies with intestine-specific induction or not (unind hemiz controls) of strong AOX expression, cultured at the indicated diet. empty and strong AOX, progeny of $U A S$-empty ${ }^{2 n d} 16$ and $U A S-A O X^{F 6}$, respectively, with $w^{1118} . d r m>$ empty and $d r m>$ strong AOX, progeny of $U A S-e m p t y^{2 n d} 16$ and $U A S-A O X^{F 6}$, respectively, with $d r m G A L 4$. The data represent averages of two or three experiments, and the error bars represent standard deviations. C, Food consumption was quantitated as described in the Materials and Methods, and the data was normalized by the relative amount of food consumed by the uninduced control larvae cultured in standard diet, which was arbitrarily set to 1.0. $d a>$ strong AOX, progeny of $U A S-A O X^{F 6}$ and $d a G A L 4$; unind hemiz control, progeny of $U A S-A O X^{F 6}$ and $w^{1118}$. * indicates significant differences between AOX-expressing and control flies, according to a two-way ANOVA. 
Figure 4. The combination of strong ubiquitous expression of AOX and low nutrient diet promotes a dramatic decrease in larval body mass. Wet (A), dry (B) and lean (C) masses of wandering L3 larvae cultured on the indicated diet were measured using an ultra-analytical balance, as described in the Materials and Methods. Total fat (D) and total protein (E) were estimated by ether extraction and the Bradford method, respectively, as described in the Materials and Methods. unind hemiz control, progeny of $U A S-A O X^{F 6}$ and $w^{1118} ; d a>$ strong AOX, progeny of $U A S-A O X^{F 6}$ and $d a G A L 4$. a, b and c represent significantly different statistical classes, according to an one-way ANOVA, followed by the Tukey post-hoc test.

Figure 5. The interaction between strong ubiquitous expression of AOX and low nutrient (LN) diet promotes OXPHOS downregulation and a starvation transcriptional response at the pupal stage. The heatmaps show changes in the levels of pupal transcripts encoding OXPHOS subunits (A) and of transcripts related to starvation-mediated response and the Insulin/Insulin-like growth factor-1 Signaling (IIS) pathway, the JAnus Kinase/Signal Transducer and Activator of Transcription (JAK/STAT) signaling pathway, and the Jun N-terminal kinase (JNK) signaling pathway (B). CI, complex I; CII, complex II; CIII, complex III; CIV, complex IV. 1-3 respectively indicate transcripts significantly $(\mathrm{p}<0.05)$ altered by the LN diet, AOX expression and the LN-AOX interaction, according to a multifactorial analysis performed using RNA-seq data and the software edge $\mathrm{R}^{77}$. The columns LN, AOX and LN-AOX show respectively color representations of the $\log _{2}$ (fold change) values of the indicated transcript in control pupae cultured in LN diet, AOX-expressing pupae cultured in standard (SD) diet, and AOX-expressing pupae culture in LN diet, each in a pairwise comparison with control pupae cultured in SD diet. 
Control pupae, progeny of $U A S-A O X^{F 6}$ and $w^{1118}$; AOX-expressing pupae, progeny of $U A S$ $A O X^{F 6}$ and $d a G A L 4$.

Figure 6. Larval amino acid levels are differentially affected by the low nutrient (LN) diet and by the strong ubiquitous expression of AOX. The columns LN, AOX and LN-AOX show respectively color representations of the $\log _{2}$ (fold change) values of the indicated metabolite in control larvae cultured in LN diet, AOX-expressing larvae cultured in standard (SD) diet, and AOX-expressing larvae culture in LN diet, each in a pairwise comparison with control larvae cultured in SD diet. 1-3 respectively indicate metabolites significantly $(p<0.05)$ altered by the LN diet, AOX expression and the LN-AOX interaction, according to a multifactorial analysis performed using mass-spectrometry data, as described in Materials and Methods. The metabolites were selected from the data shown in the Supplemental Table S18, and were clustered according to functional categories or the most frequent metabolic pathway in which they participate. TCA, tricarboxilic acid cycle; Pent. Phos., pentose phosphate pathway; Inositol deg., inositol degradation pathway. Control larvae, progeny of $w^{1118}$ and $d a G A L 4$; AOXexpressing larvae, progeny of $U A S-A O X^{F 6}$ and $d a G A L 4$.

Figure 7. Rescue of the lethality of AOX-expressing pupae cultured on LN diet by methionine/tryptophan supplementation. Pupal viability of control (unind hemiz controls, progeny of $U A S-A O X^{F 6}$ and $\left.w^{1118}\right)$ and strong ubiquitously-driven AOX-expressing (da>strong AOX, progeny of $U A S-A O X^{F 6}$ and $d a G A L 4$ ) flies cultured at the indicated diet, supplemented with methionine and/or tryptophan (A), or proline and/or glutamate (B). The “-”" indicates no supplementation. The final concentrations of extra methionine added in the "+ met" experiments 
were $0.18,0.35$ and $0.7 \mathrm{mM}$, and in the "+ met \& trp" experiments were 0.35 and $0.7 \mathrm{mM}$. The final concentrations of extra tryptophan in the "+ trp" experiments were $0.1,0.2$ and $0.4 \mathrm{mM}$, and in the "+ met \& trp" experiments were 0.2 and $0.4 \mathrm{mM}$. The final concentration of extra proline and glutamate used in the "+ pro", "+ glu" and "+ pro \& glu" were 1.7 and $2.9 \mathrm{mM}$, respectively. These concentrations were selected based on previously published work ${ }^{79}$.

Figure 8. Lower (intermediate) levels of ubiquitous AOX expression promotes larval growth. Larvae of the fly line $3 \mathrm{x} t u b A O X^{3}$, which expresses AOX $\sim 5$ fold lower than the progeny of $U A S$ $A O X^{F 6}$ and $d a G A L 4^{24}$, and larvae of the control line $w^{1118}$ were cultured on standard diet. A, Light microscopy images of representative larvae. B, Quantitation of larval wet and dry masses. C, Quantitation of larval length and width. The values indicate averages of at least 25 individual measurements, and the error bars represent standard deviations. * indicates statistical differences $(\mathrm{p}<0.001)$ according to a Student's t-test. $\mathrm{a}, \mathrm{b}$ and $\mathrm{c}$ represent significantly different statistical classes, according to an one-way ANOVA, followed by the Tukey post-hoc test.

Figure 9. AOX expression during Drosophila development. A, Summary of the results presented in this work. B, Proposed model of how AOX influences larval growth and biomass accumulation, which may impact pupal viability and adult eclosion rates. See text for details. 
A

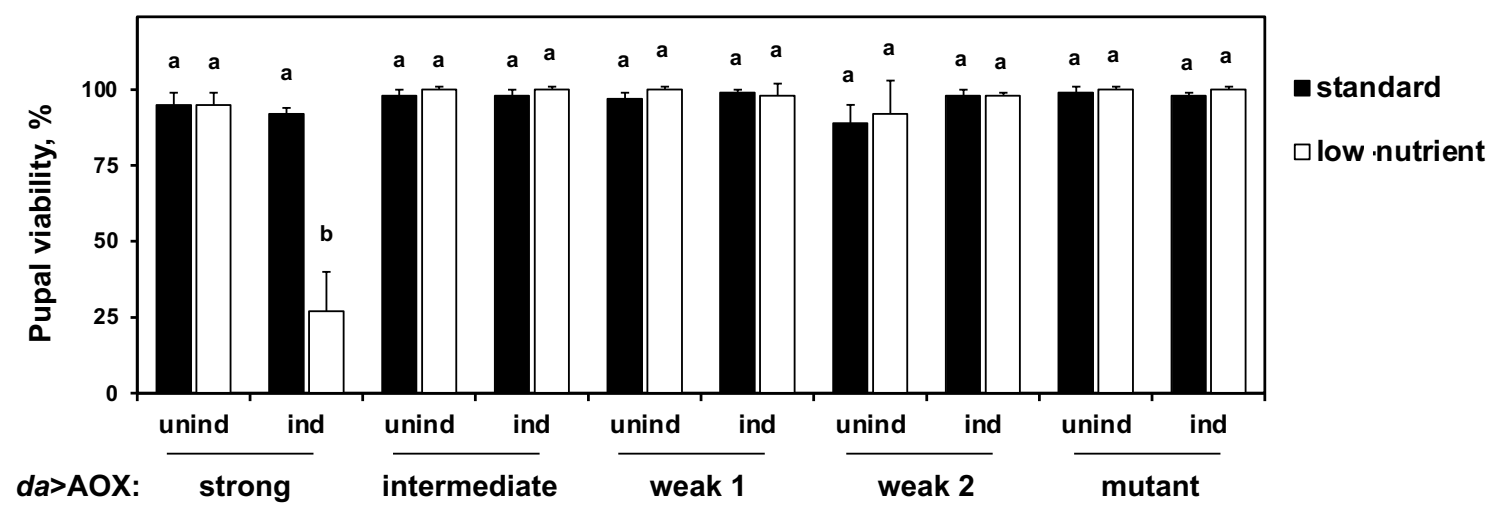

B

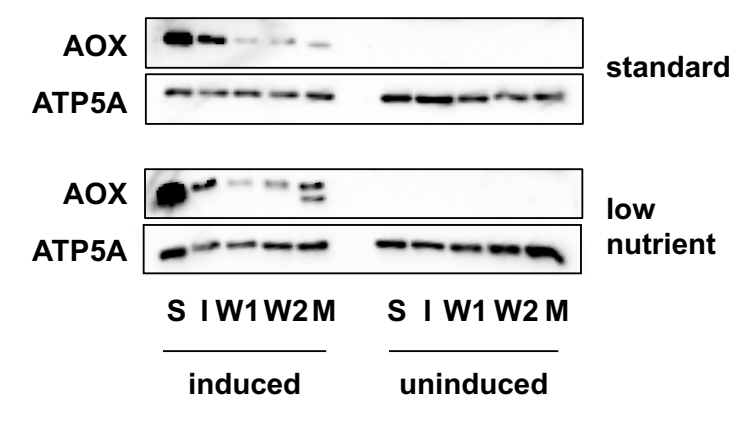

C

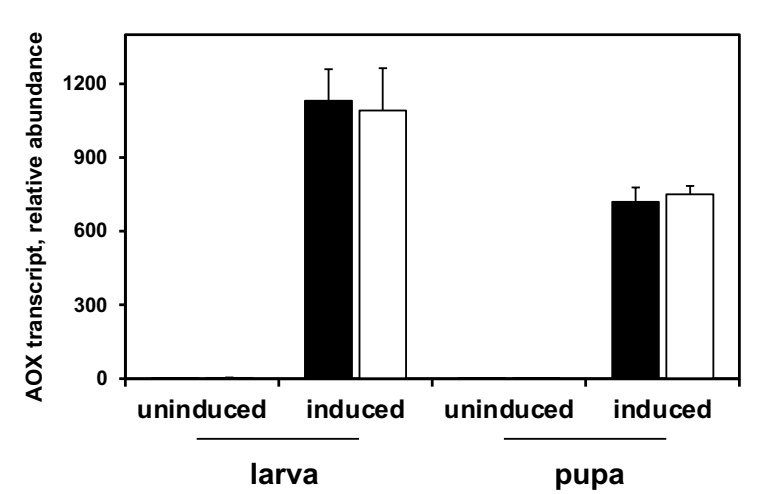

D

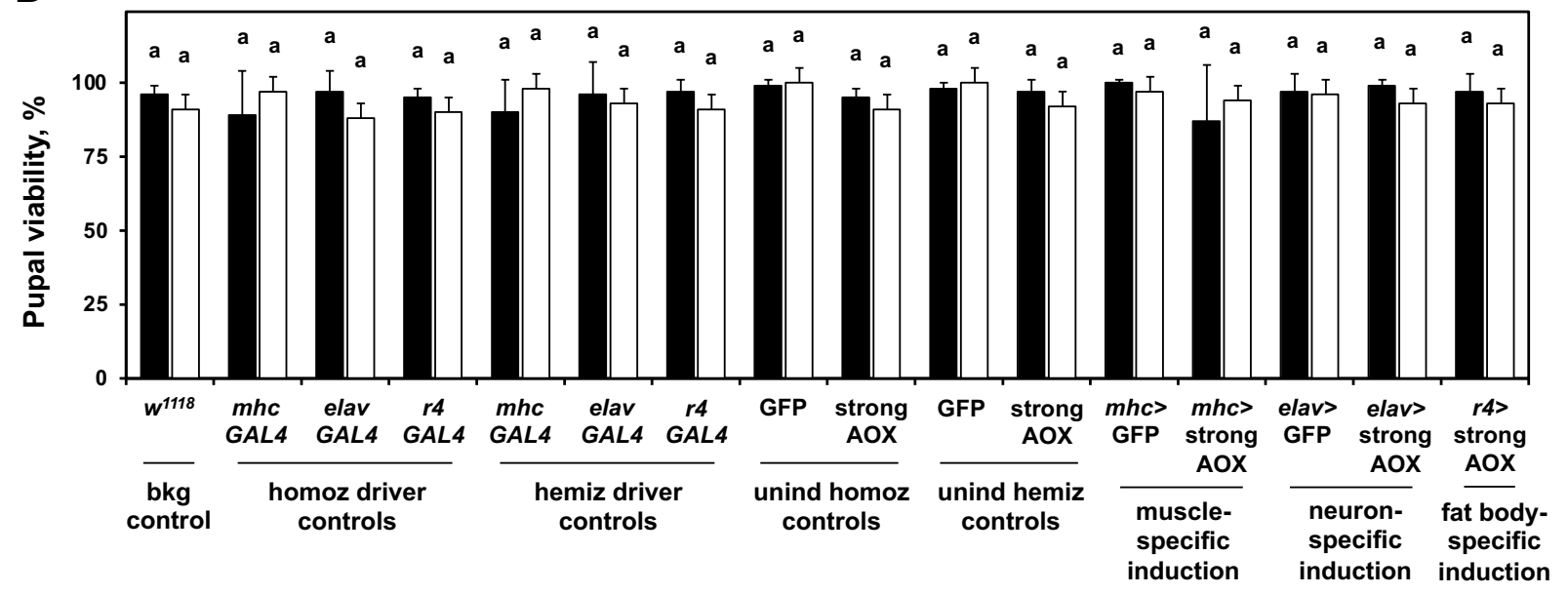

Figure 1. 
A

Proteolysis
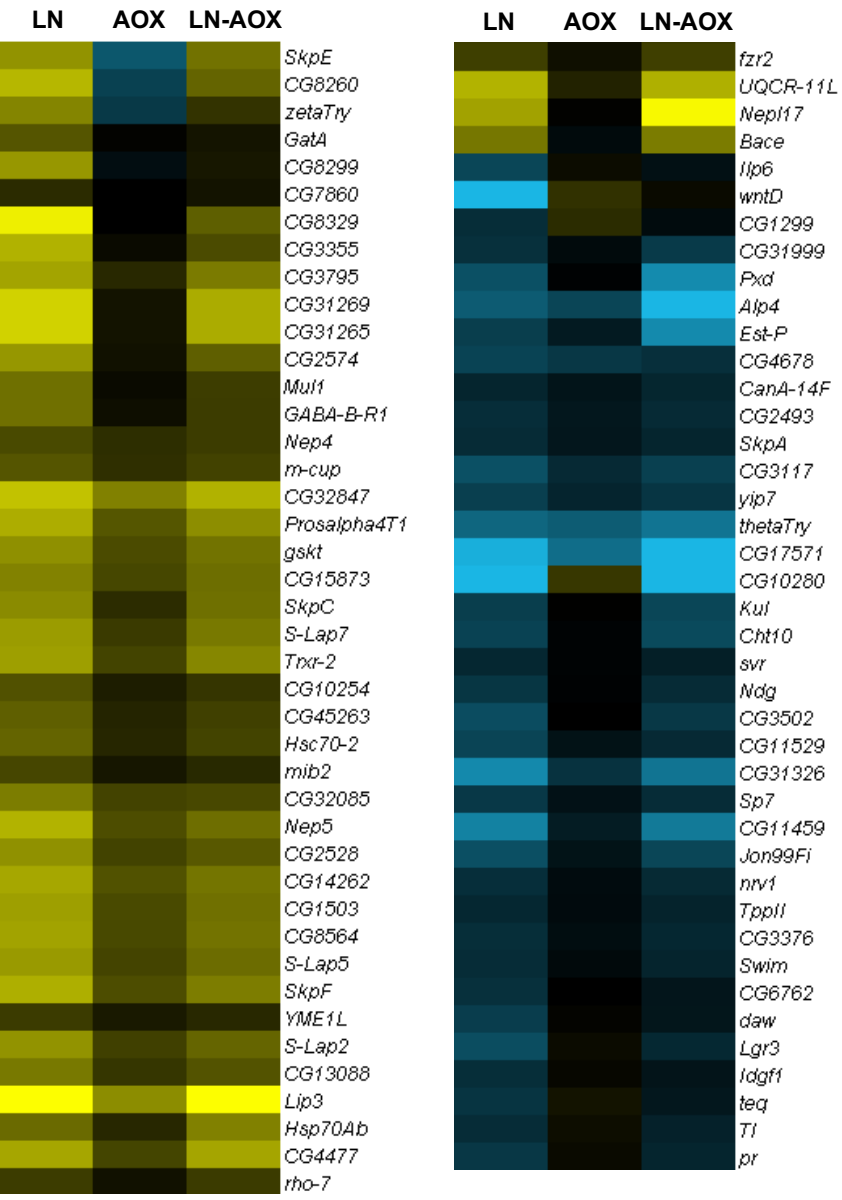

Chitin metabolism

LN AOX LN-AOX
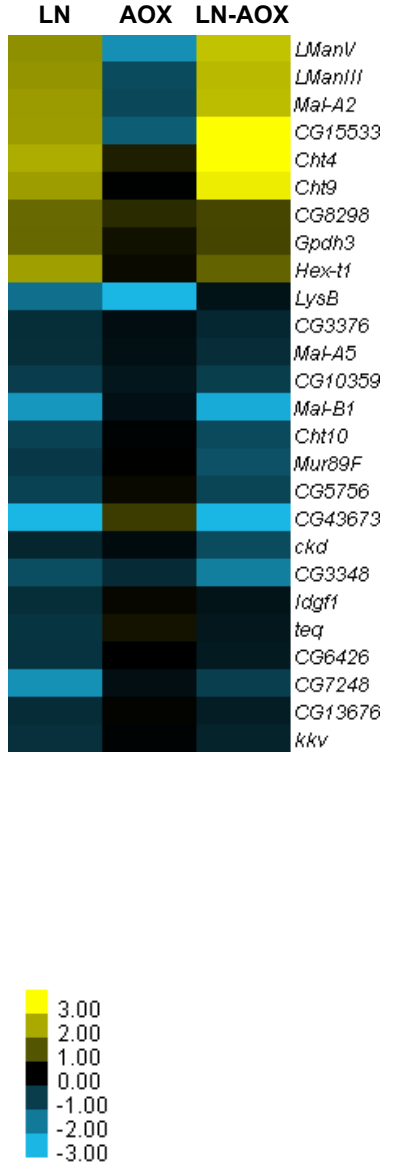

B

Proteolysis

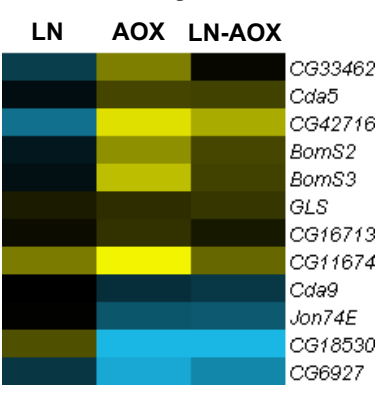

C

\section{Proteolysis}

LN AOX LN-AOX

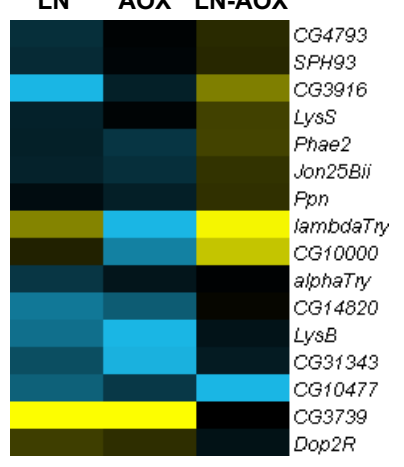

Chitin metabolism

LN AOX LN-AOX

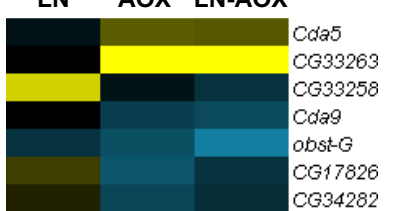

Chitin metabolism

LN AOX LN-AOX

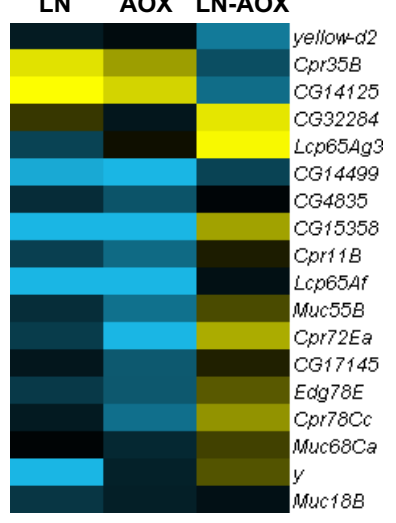

Figure 2. 
A

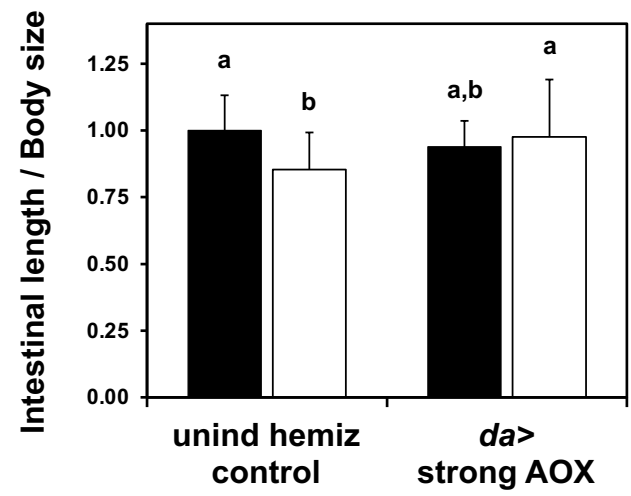

C

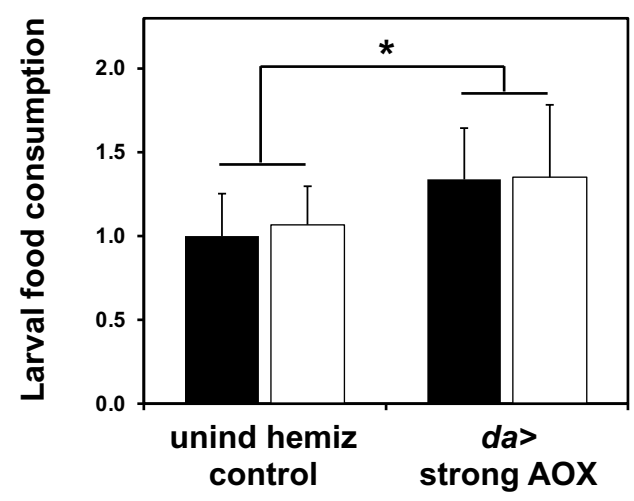

B

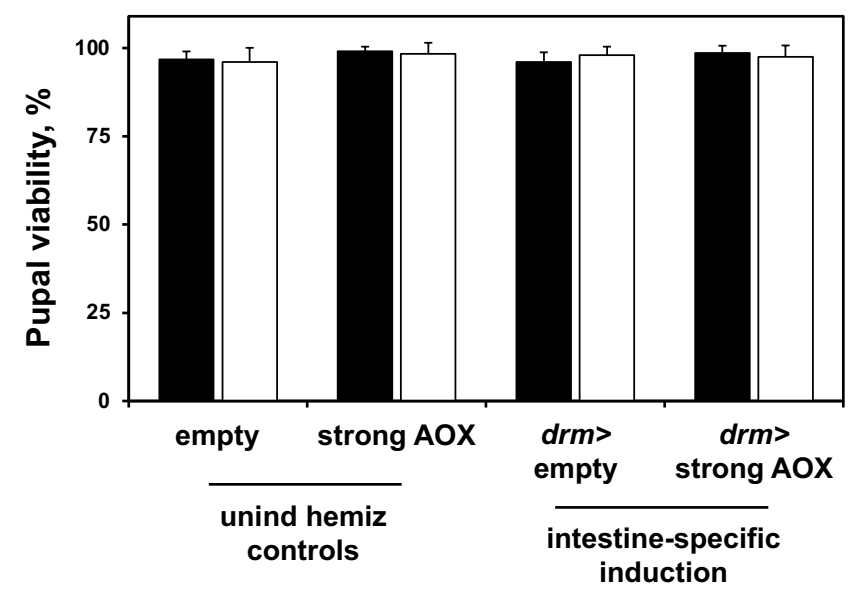

standard

$\square$ low nutrient

Figure 3. 

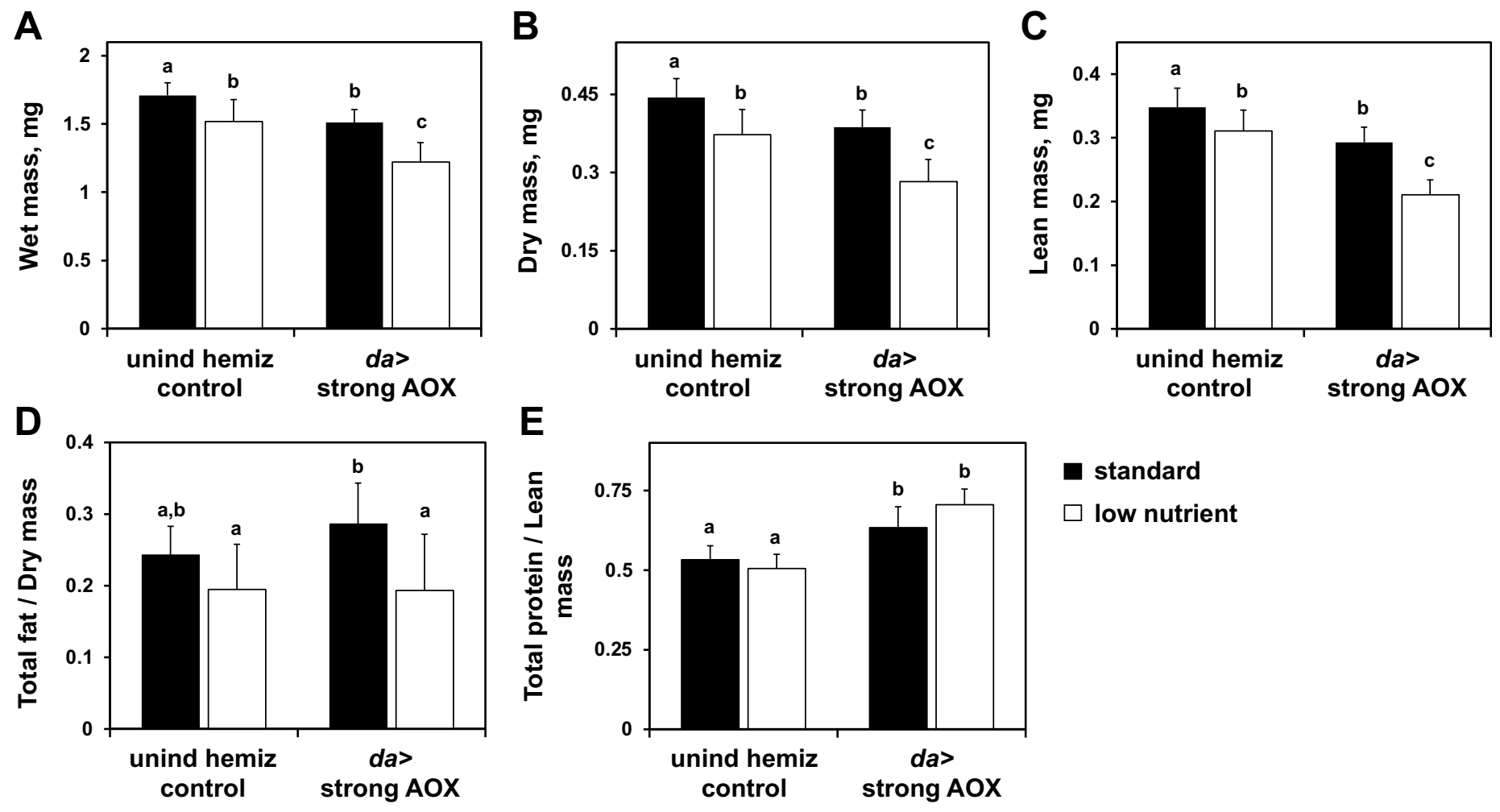

Figure 4. 
A

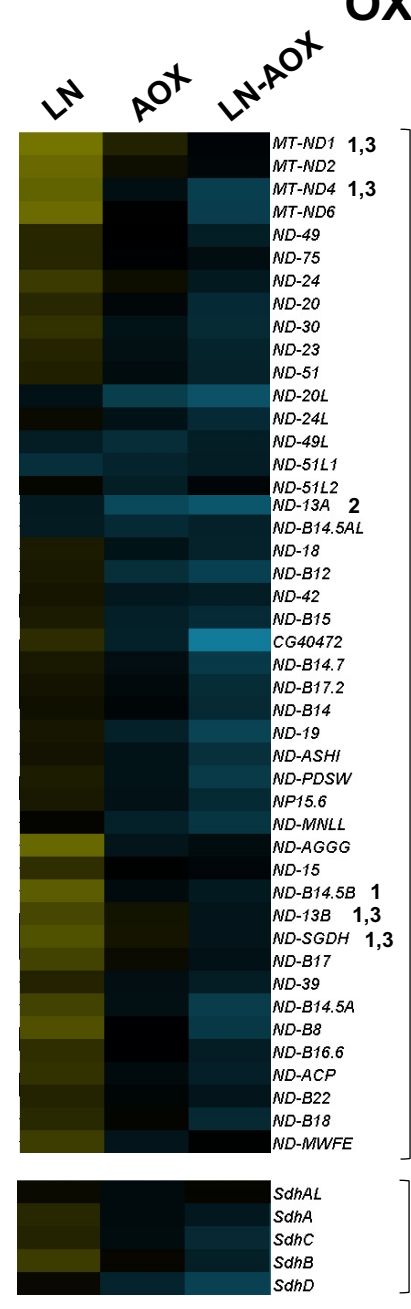

OXPHOS

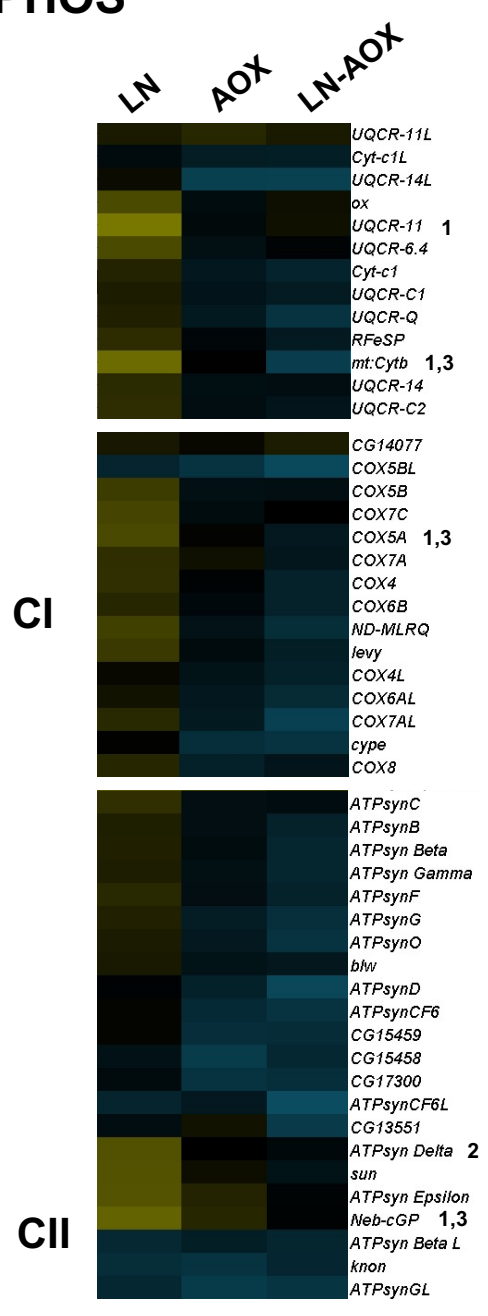

B

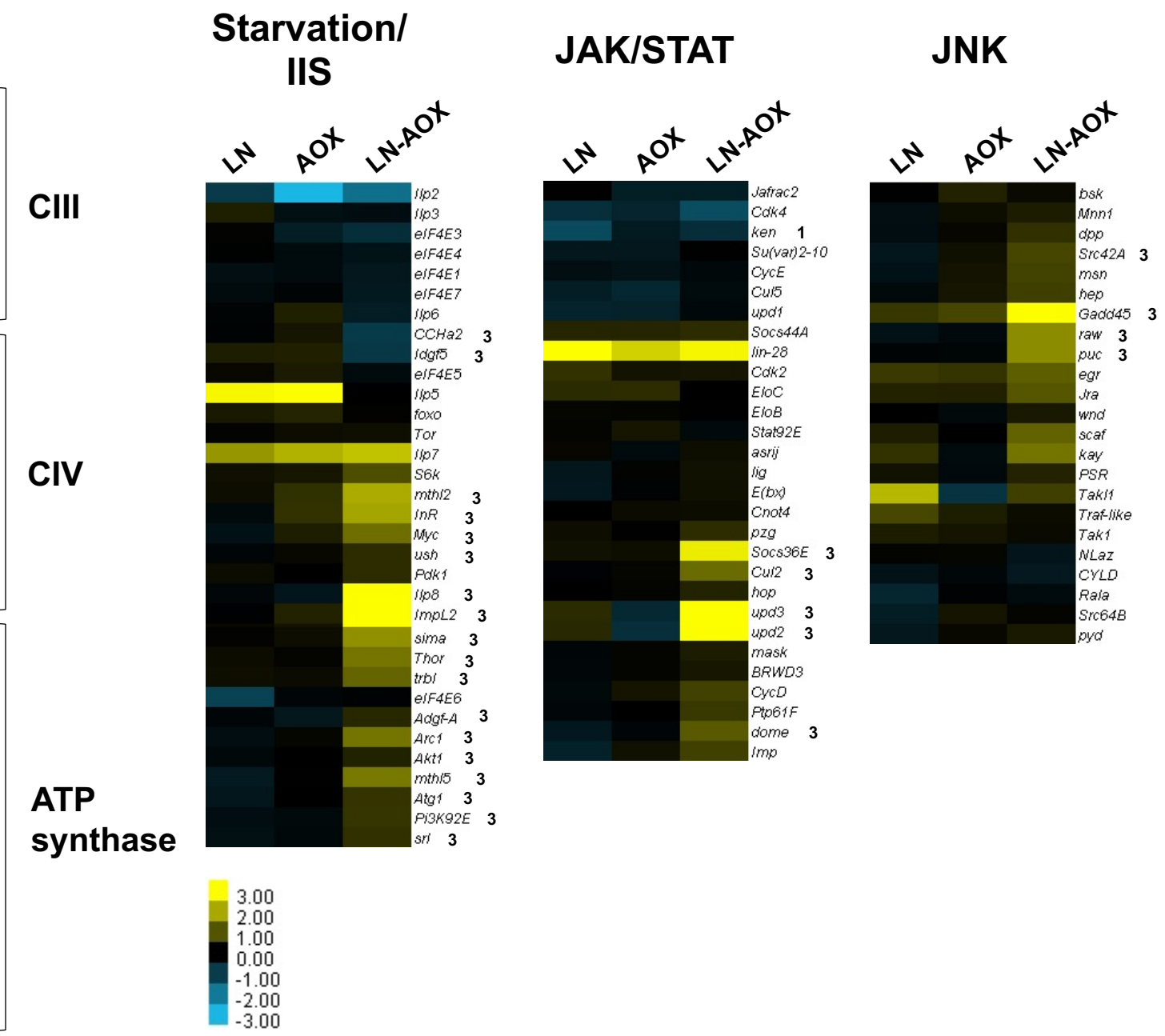

Figure 5. 


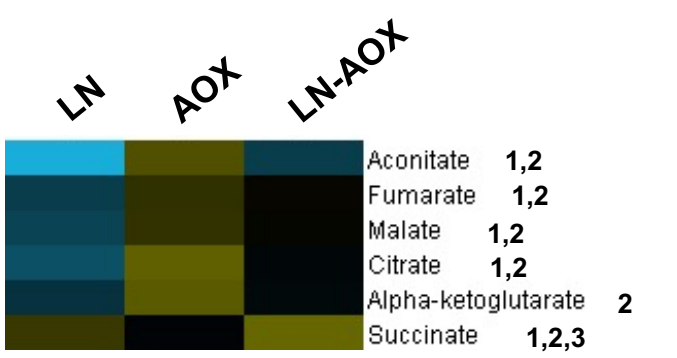

TCA cycle

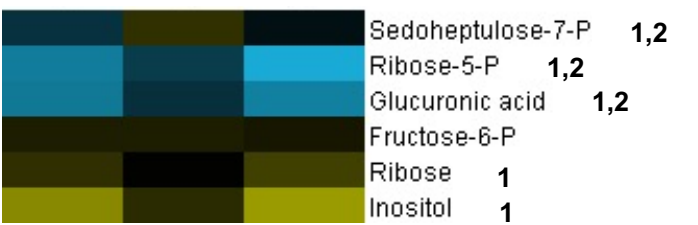

Pent. Phosp.I Inositol deg.

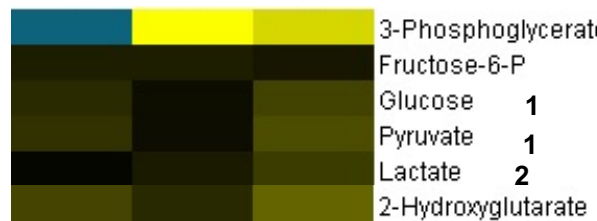

$1,2,3$

Glucose metabolism

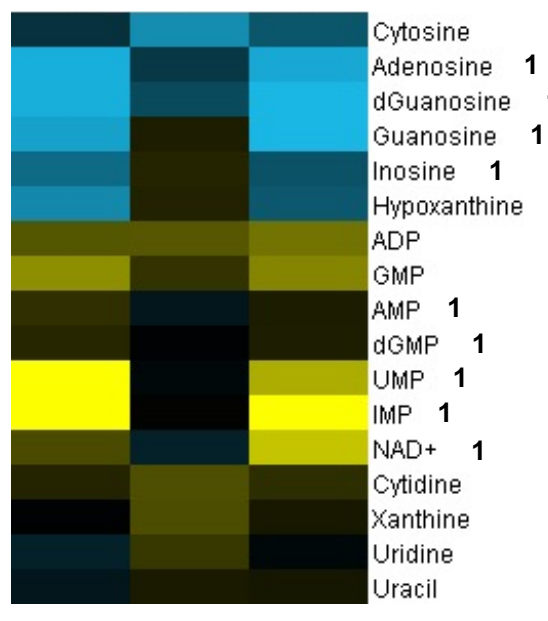

Nucleotides, Nucleosides

\& Bases

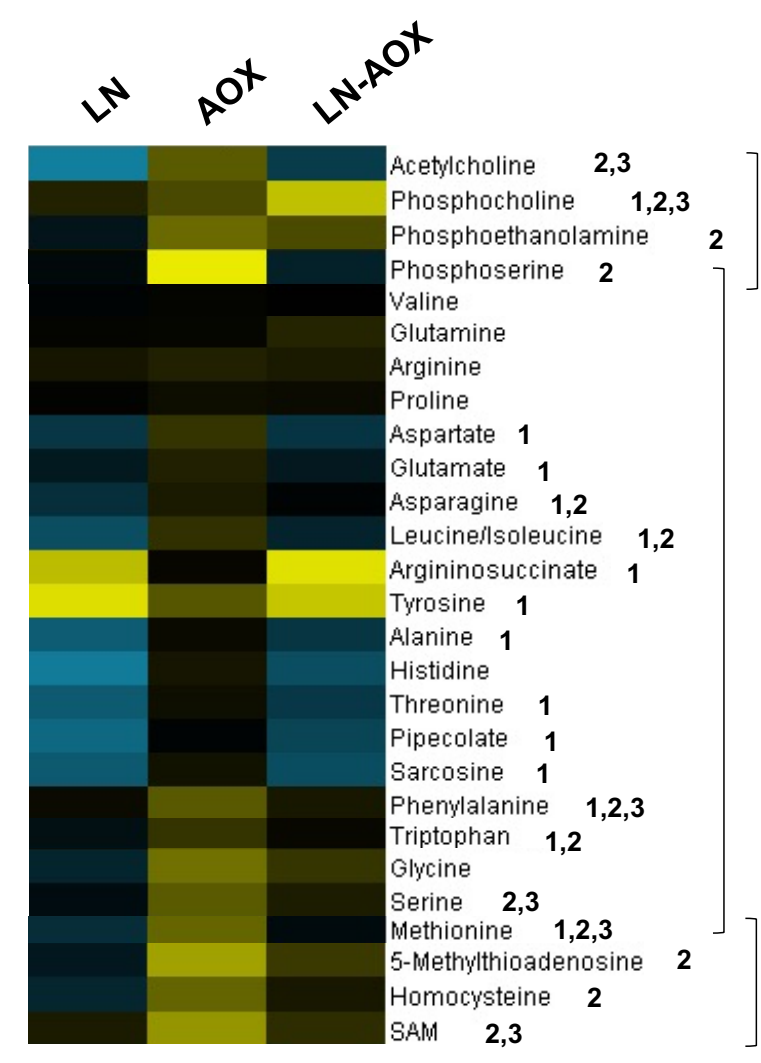

Amino acids
Choline/ Phospholipid metabolites

Methionine cycle

Figure 6. 
A

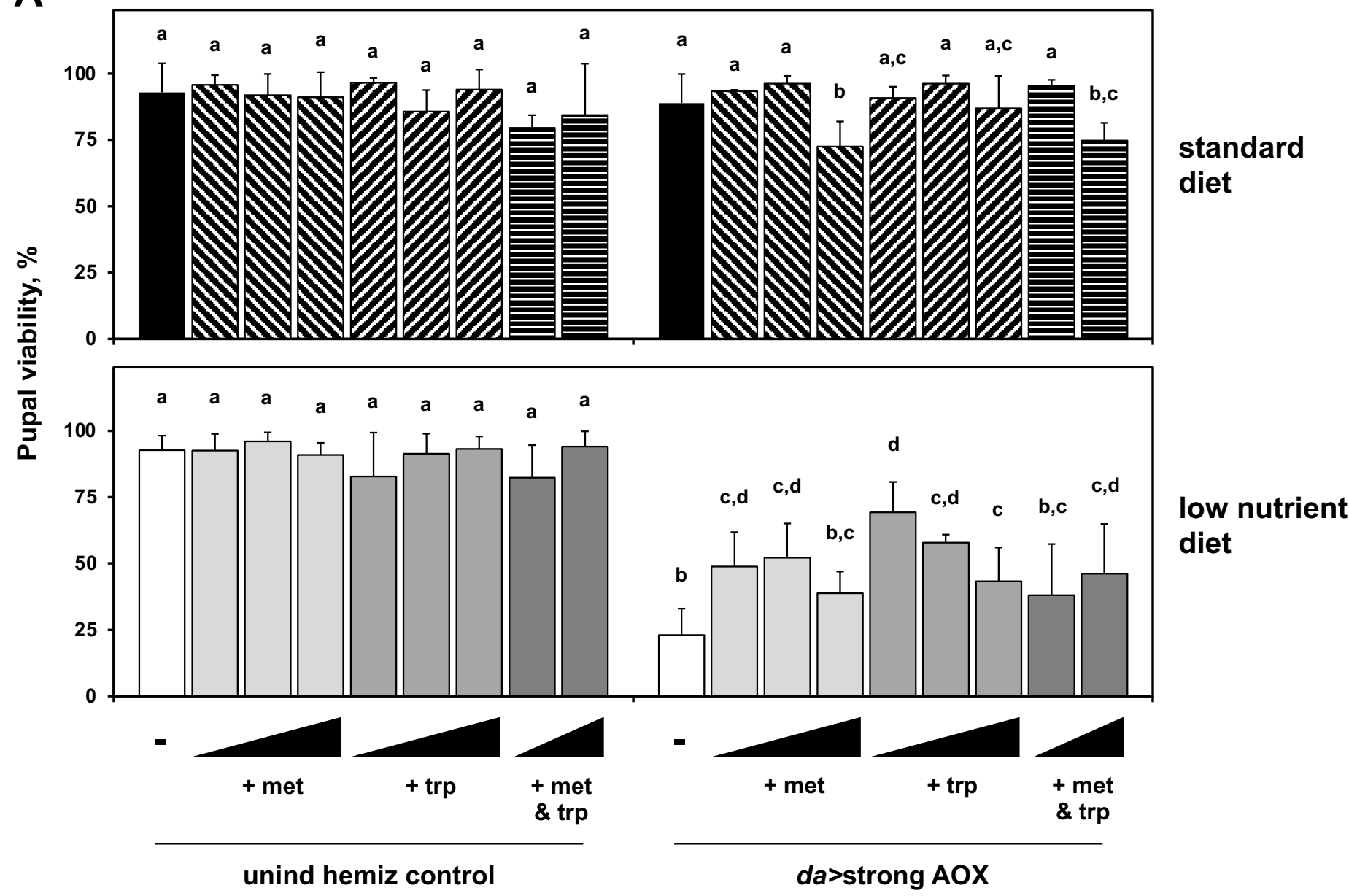

B

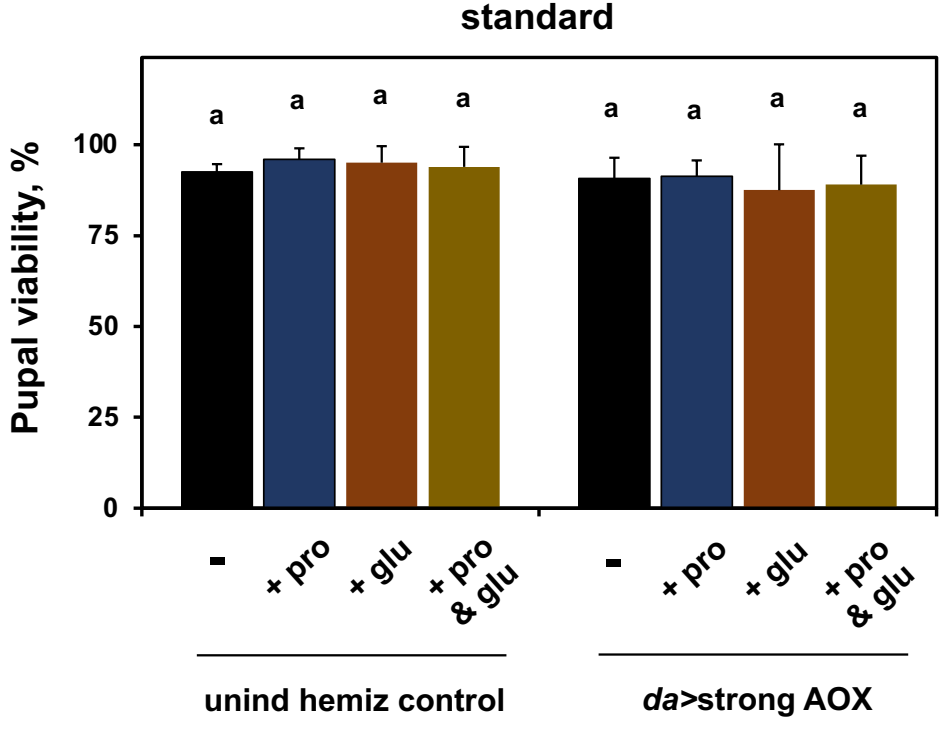

low nutrient

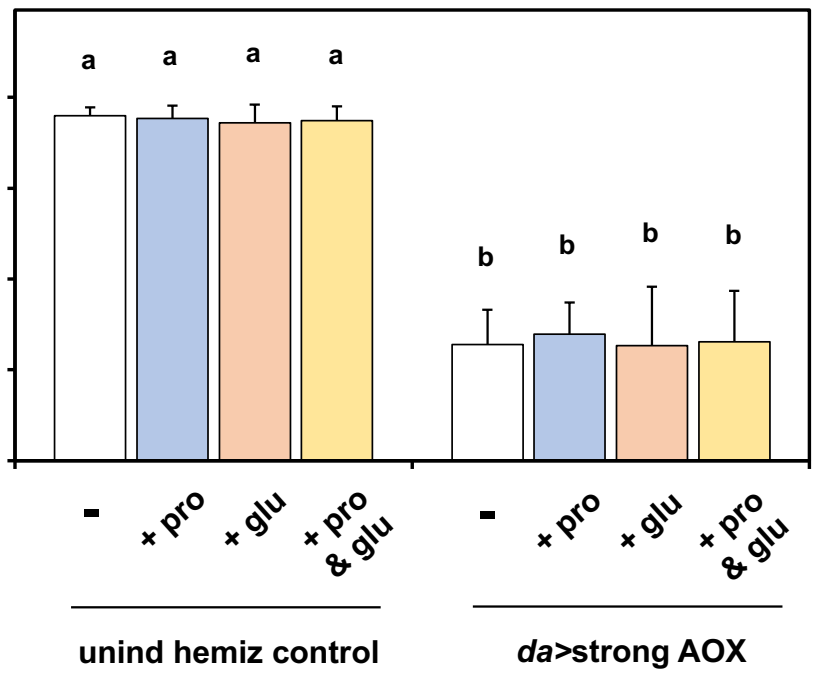

Figure 7. 
bioRxiv preprint doi: https://doi.org/10.1101/2021.09.24.461559; this version posted September 24, 2021. The copyright holder for this preprint (which was not certified by peer review) is the author/funder, who has granted bioRxiv a license to display the preprint in perpetuity. It is made available under aCC-BY-NC-ND 4.0 International license.

A

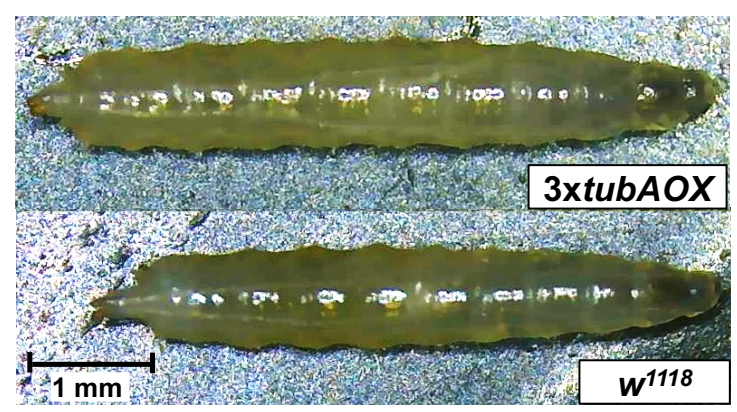

C

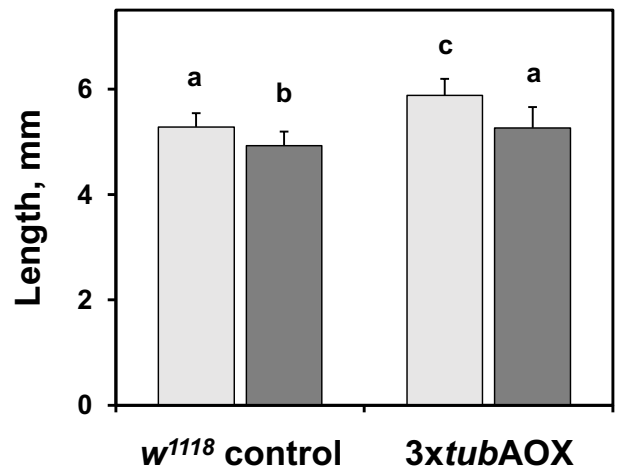

B
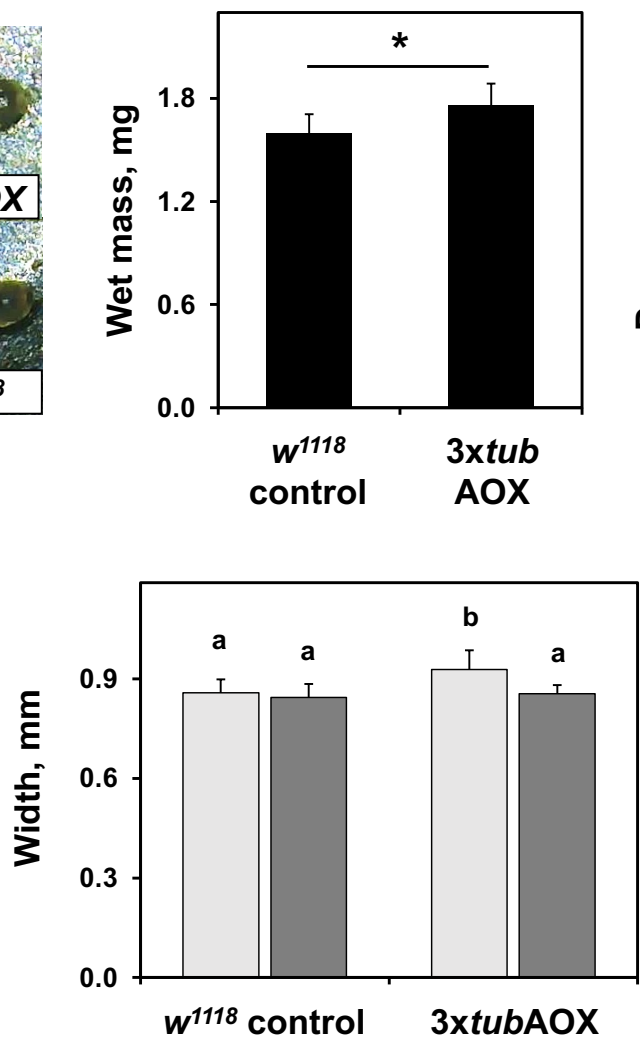

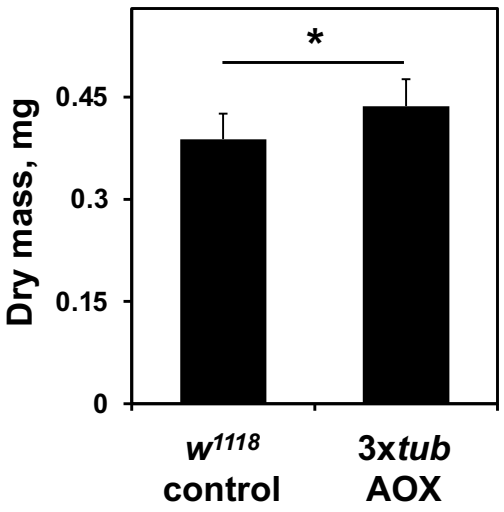

$\square$ females

$\square$ males

Figure 8. 


\section{AOX in development}

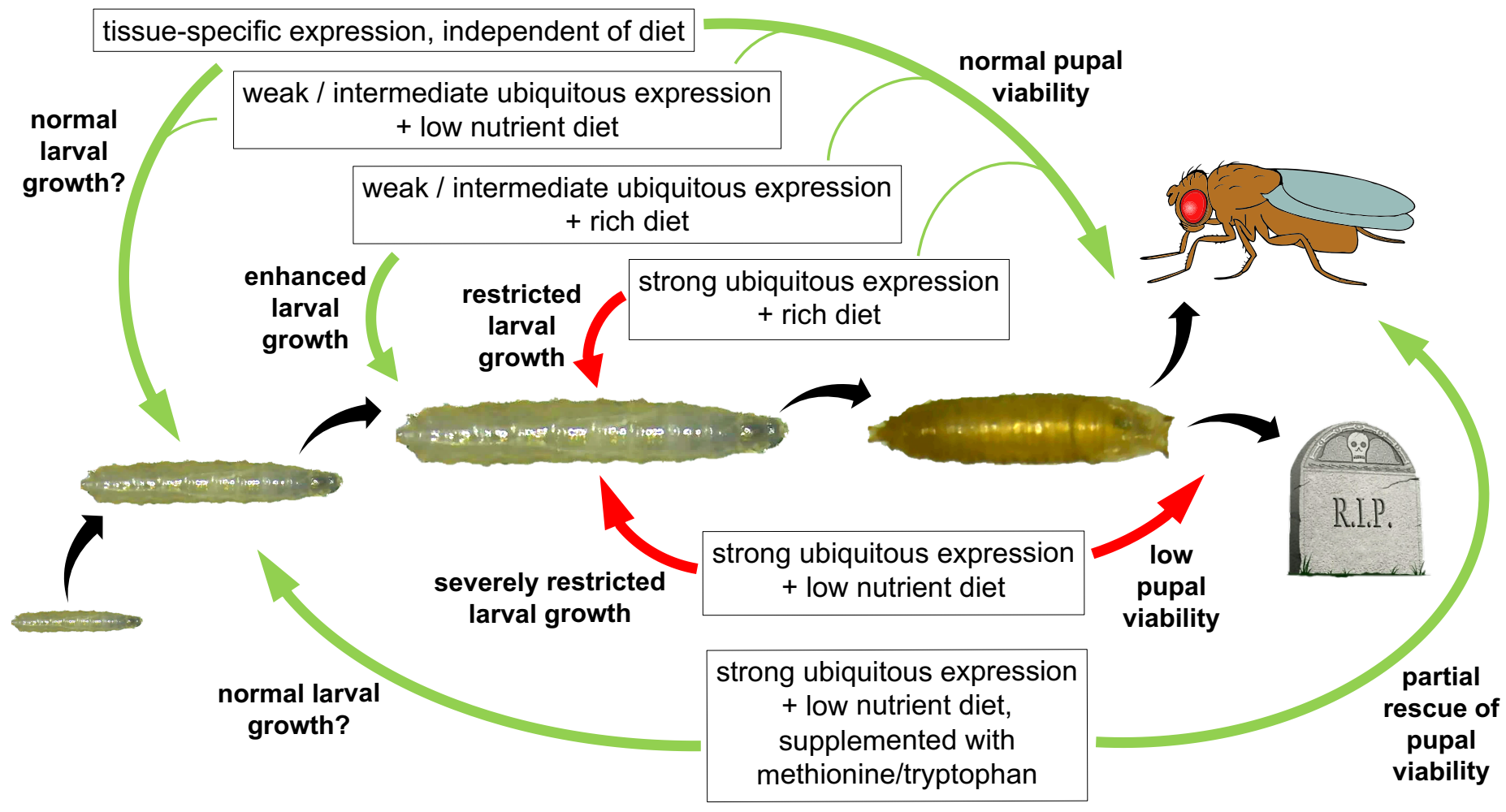

B

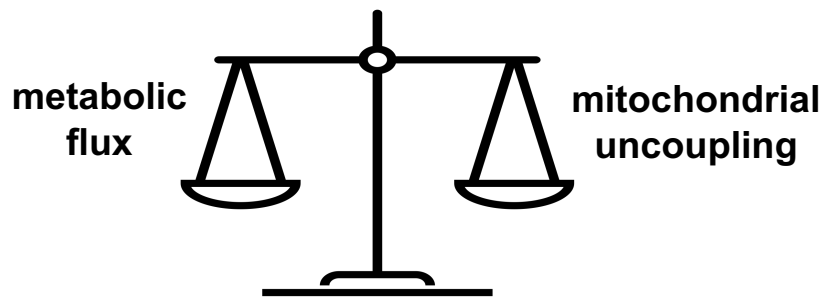

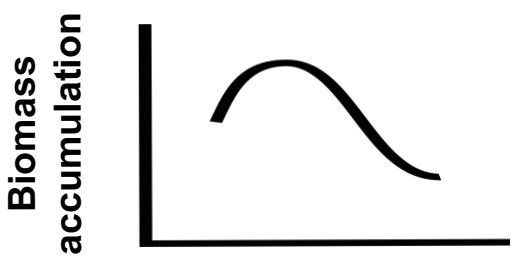

AOX level

\section{Figure 9.}

